\title{
An Assessment of SAPT and Supermolecular EDAs Approaches for the Development of Separable and Polarizable force fields
}

Sehr Naseem-Khan, ${ }^{\dagger}$ Nohad Gresh, ${ }^{\dagger}$ Alston J. Misquitta,,${ }^{* \dagger}$ and Jean-Philip

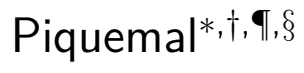

$\dagger$ Laboratoire de Chimie Théorique, Sorbonne Université, UMR 7616 CNRS, 75005, Paris, France

$\ddagger$ School of Physics and Astronomy and the Thomas Young Centre for Theory and Simulation of Materials at Queen Mary University of London, London E1 4NS, U.K. IInstitut Universitaire de France, 75005, Paris, France

$\S$ The University of Texas at Austin, Department of Biomedical Engineering, TX, USA

E-mail: a.j.misquitta@qmul.ac.uk; jean-philip.piquemal@sorbonne-universite.fr 


\section{Supporting Information Available}

\section{Contents}

1 Accuracy of the methods: Auxiliary data 3

$1.1 \mathrm{Cl}^{-} \ldots \mathrm{H}_{2} \mathrm{O} \quad \& \mathrm{OH}^{-} \ldots \mathrm{H}_{2} \mathrm{O} \quad \ldots \ldots \ldots$

1.2 Comments on the use of the asymptotic correction for anions . . . . . . . . 5

2 Role of the $S^{2}$ approximation: Auxiliary data $r$

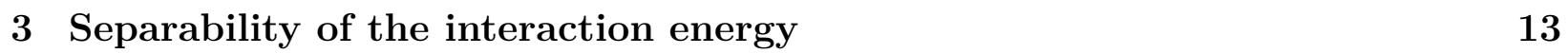

3.1 Auxiliary plots . . . . . . . . . . . . . . . . . . . 13

3.2 Comparison of EDAs: ALMO, RVS \& CSOV . . . . . . . . . . . . . 15

4 Polarization models $\quad 17$

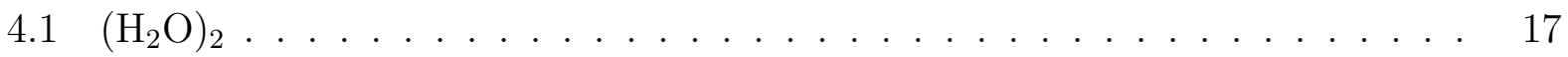

$4.2 \mathrm{Zn}^{2+} \ldots \mathrm{H}_{2} \mathrm{O} \ldots \ldots \ldots \ldots \ldots$

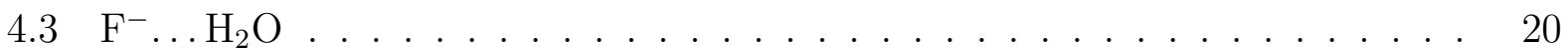

5 Sensitivity of $E_{\text {elst }}^{(1)}+E_{\text {exch }}^{(1)}$ and $E_{\mathrm{CD}}$ to the wavefunction 22

6 Recommendations for SAPT(DFT) calculations $\quad 24$

$\begin{array}{llr}7 & \text { Energy tables } & 27\end{array}$

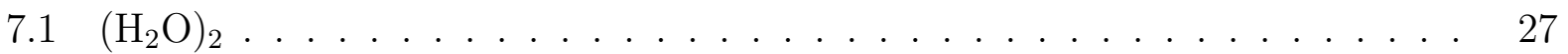

$7.2 \mathrm{Zn}^{2+} \ldots \mathrm{H}_{2} \mathrm{O} \ldots \ldots \ldots \ldots$

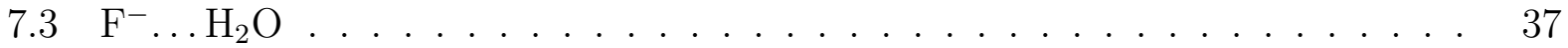

$7.4 \mathrm{Cl}^{-} \ldots \mathrm{H}_{2} \mathrm{O} \ldots \ldots \ldots \ldots \ldots \ldots$

$7.5 \mathrm{OH}^{-} \ldots \mathrm{H}_{2} \mathrm{O} \ldots \ldots \ldots \ldots \ldots \ldots$ 
8 Complexes cartesian coordinates $\quad 48$

$8.1 \quad\left(\mathrm{H}_{2} \mathrm{O}\right)_{2} \ldots \ldots \ldots \ldots \ldots \ldots$

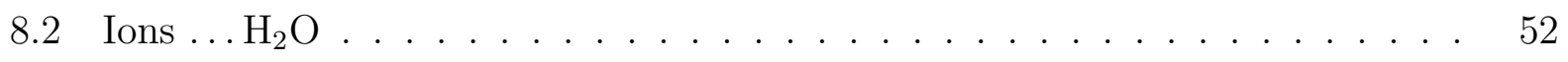

$\begin{array}{ll}\text { References } & 54\end{array}$

1 Accuracy of the methods: Auxiliary data

$1.1 \mathrm{Cl}^{-} \ldots \mathrm{H}_{2} \mathrm{O} \& \mathrm{OH}^{-} \ldots \mathrm{H}_{2} \mathrm{O}$

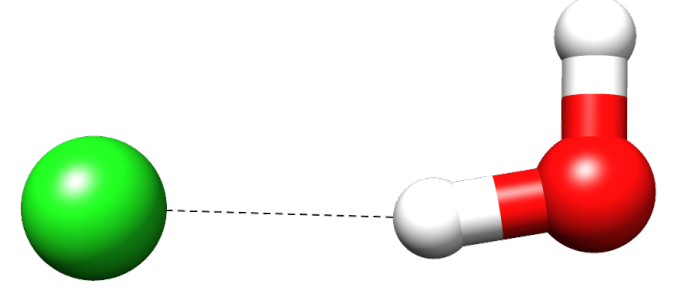

(a)

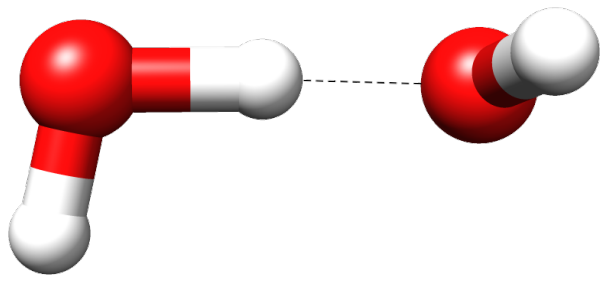

(b)

Figure S1: Representation of : (a) $\mathrm{Cl}^{-} \ldots \mathrm{H}_{2} \mathrm{O}$ and (b) $\mathrm{OH}^{-} \ldots \mathrm{H}_{2} \mathrm{O}$ complexes. 

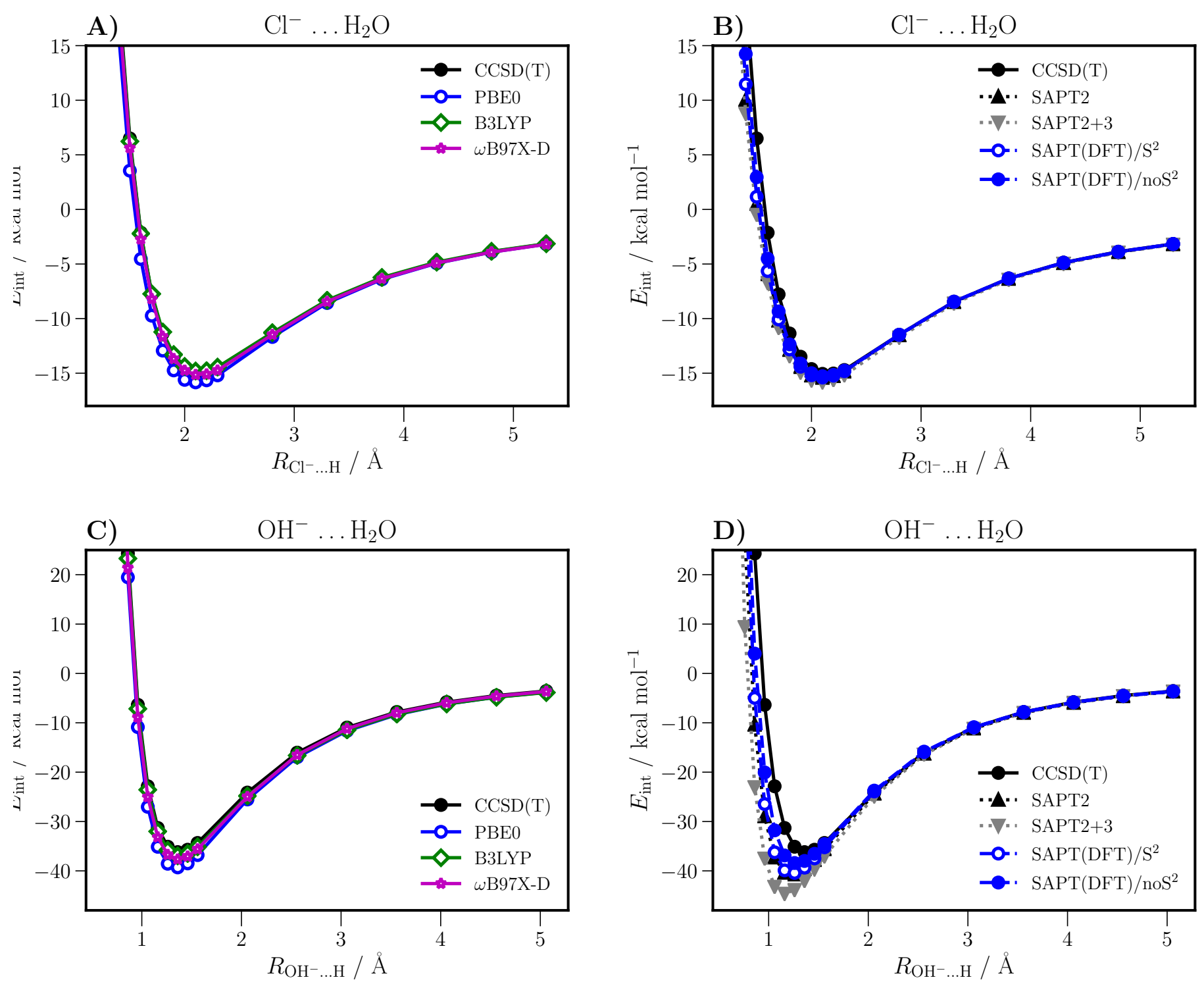

Figure S2: Comparison of the intermolecular interaction energy between DFT-based supermolecular EDAs, SAPT models, SAPT(DFT) and $\mathrm{CCSD}(\mathrm{T})$ for the $\mathrm{Cl}^{-} \ldots \mathrm{H}_{2} \mathrm{O}$ and $\mathrm{OH}^{-}$ $\ldots \mathrm{H}_{2} \mathrm{O}$ complexes. The asymptotically corrected PBE0 functional is used for SAPT(DFT). The $S^{2}$ approximation is used for the second and third-order exchange energies in the SAPT models and $\mathrm{SAPT}(\mathrm{DFT}) / S^{2}$. For $\mathrm{SAPT}(\mathrm{DFT}) / \mathrm{no} S^{2}$ this approximation is present only in the $E_{\text {disp,exch }}^{(2)}$ energy. 


\subsection{Comments on the use of the asymptotic correction for anions}

Table S1: Influence of the Asymptotic Correction (AC) on anionic-water complexes $^{1}$ in SAPT(DFT). First, the AC was applied on both anion and water molecule (AC). Then, the AC was kept on the water molecule but was removed for the anion (noAC). PBE0 functional was used for SAPT(DFT) calculations with the aug-cc-pVTZ basis set. For SAPT(DFT) calculations, the $S^{2}$ approximation is only used for the $E_{\text {disp,exch }}^{(2)}$ energy. Values in $\mathrm{kcal} \mathrm{mol}^{-1}$.

\begin{tabular}{|c|c|c|c|c|c|c|c|c|c|c|c|}
\hline \multicolumn{6}{|c|}{$E_{\text {int }}: \mathrm{F}^{-} \ldots \mathrm{H}_{2} \mathrm{O}$} & \multicolumn{6}{|c|}{$E_{\text {int }}: \mathrm{Cl}^{-} \ldots \mathrm{H}_{2} \mathrm{O}$} \\
\hline$d(\AA)$ & $\mathrm{AC}$ & noAC & SAPT2 & SAPT $2+3$ & $\operatorname{CCSD}(\mathrm{T})$ & $\mathrm{d}(\AA)$ & $\mathrm{AC}$ & noAC & SAPT2 & SAPT $2+3$ & $\operatorname{CCSD}(\mathrm{T})$ \\
\hline 0.70 & 132.85 & 133.78 & 107.15 & 89.08 & 161.48 & 1.30 & 31.11 & 30.17 & 24.04 & 22.40 & 38.53 \\
\hline 0.80 & 53.46 & 54.48 & 36.86 & 24.96 & 70.72 & 1.40 & 14.24 & 13.56 & 10.05 & 8.77 & 19.41 \\
\hline 0.90 & 11.82 & 12.88 & 1.31 & -6.60 & 21.67 & 1.50 & 2.95 & 2.50 & 0.51 & -0.48 & 6.50 \\
\hline 1.00 & -10.31 & -9.24 & -16.95 & -22.35 & -5.16 & 1.60 & -4.51 & -4.79 & -5.93 & -6.73 & -2.13 \\
\hline 1.10 & -21.98 & -20.90 & -26.23 & -30.04 & -19.70 & 1.70 & -9.34 & -9.49 & -10.19 & -10.84 & -7.78 \\
\hline 1.20 & -27.81 & -26.74 & -30.62 & -33.40 & -27.20 & 1.80 & -12.36 & -12.40 & -12.87 & -13.43 & -11.36 \\
\hline 1.30 & -30.30 & -29.28 & -32.26 & -34.34 & -30.62 & 1.90 & -14.12 & -14.08 & -14.44 & -14.93 & -13.49 \\
\hline 1.40 & -30.84 & -29.88 & -32.29 & -33.88 & -31.61 & 2.00 & -15.00 & -14.91 & -15.21 & -15.65 & -14.62 \\
\hline 1.50 & -30.24 & -29.35 & -31.37 & -32.62 & -31.18 & 2.10 & -15.28 & -15.15 & -15.43 & -15.82 & -15.06 \\
\hline 1.60 & -28.99 & -28.18 & -29.91 & -30.90 & -29.94 & 2.20 & -15.15 & -15.01 & -15.26 & -15.62 & -15.03 \\
\hline 2.10 & -20.47 & -20.07 & -20.90 & -21.27 & -20.91 & 2.30 & -14.77 & -14.61 & -14.85 & -15.18 & -14.71 \\
\hline 2.60 & -13.78 & -13.64 & -14.02 & -14.17 & -13.92 & 2.80 & -11.48 & -11.36 & -11.52 & -11.72 & -11.48 \\
\hline 3.10 & -9.56 & -9.54 & -9.72 & -9.77 & -9.59 & 3.30 & -8.44 & -8.39 & -8.49 & -8.61 & -8.45 \\
\hline 3.60 & -6.93 & -6.95 & -7.05 & -7.06 & -6.93 & 3.80 & -6.32 & -6.30 & -6.36 & -6.43 & -6.32 \\
\hline 4.10 & -5.25 & -5.27 & -5.34 & -5.34 & -5.23 & 4.30 & -4.88 & -4.88 & -4.91 & -4.96 & -4.87 \\
\hline 4.60 & -4.12 & -4.13 & -4.18 & -4.18 & -4.10 & 4.80 & -3.88 & -3.88 & -3.91 & -3.95 & -3.87 \\
\hline 5.10 & -3.32 & -3.33 & -3.37 & -3.37 & -3.30 & 5.30 & -3.16 & -3.17 & -3.19 & -3.22 & -3.16 \\
\hline
\end{tabular}

\begin{tabular}{rrrrrr}
\hline \multicolumn{6}{c}{$E_{\text {int }}: \mathrm{OH}^{-} \ldots \mathrm{H}_{2} \mathrm{O}$} \\
\cline { 1 - 4 } $\mathrm{d}(\AA)$ & $\mathrm{AC}$ & noAC & $\mathrm{SAPT} 2$ & $\mathrm{SAPT} 2+3$ & $\mathrm{CCSD}(\mathrm{T})$ \\
0.66 & 143.86 & 143.74 & 106.99 & 79.07 & 183.94 \\
0.76 & 51.51 & 51.69 & 28.23 & 9.41 & 80.55 \\
0.86 & 4.02 & 4.33 & -10.40 & -23.03 & 24.31 \\
0.96 & -20.06 & -19.71 & -28.94 & -37.50 & -6.36 \\
1.06 & -31.79 & -31.42 & -37.29 & -43.23 & -22.86 \\
1.16 & -36.88 & -36.49 & -30.62 & -44.62 & -31.31 \\
1.26 & -38.41 & -37.99 & -32.26 & -43.85 & -35.11 \\
1.36 & -37.99 & -37.55 & -32.29 & -42.01 & -36.20 \\
1.46 & -36.55 & -36.09 & -31.37 & -39.65 & -35.72 \\
1.56 & -34.58 & -34.11 & -29.91 & -37.05 & -34.35 \\
2.06 & -23.74 & -23.36 & -20.90 & -24.87 & -24.14 \\
2.56 & -15.84 & -15.63 & -14.02 & -16.43 & -16.05 \\
3.06 & -10.89 & -10.81 & -9.72 & -11.20 & -10.96 \\
3.56 & -7.81 & -7.80 & -7.05 & -7.97 & -7.81 \\
4.06 & -5.84 & -5.86 & -5.34 & -5.93 & -5.81 \\
4.56 & -4.52 & -4.55 & -4.18 & -4.58 & -4.48 \\
5.06 & -3.61 & -3.63 & -3.37 & -3.64 & -3.56 \\
\hline
\end{tabular}



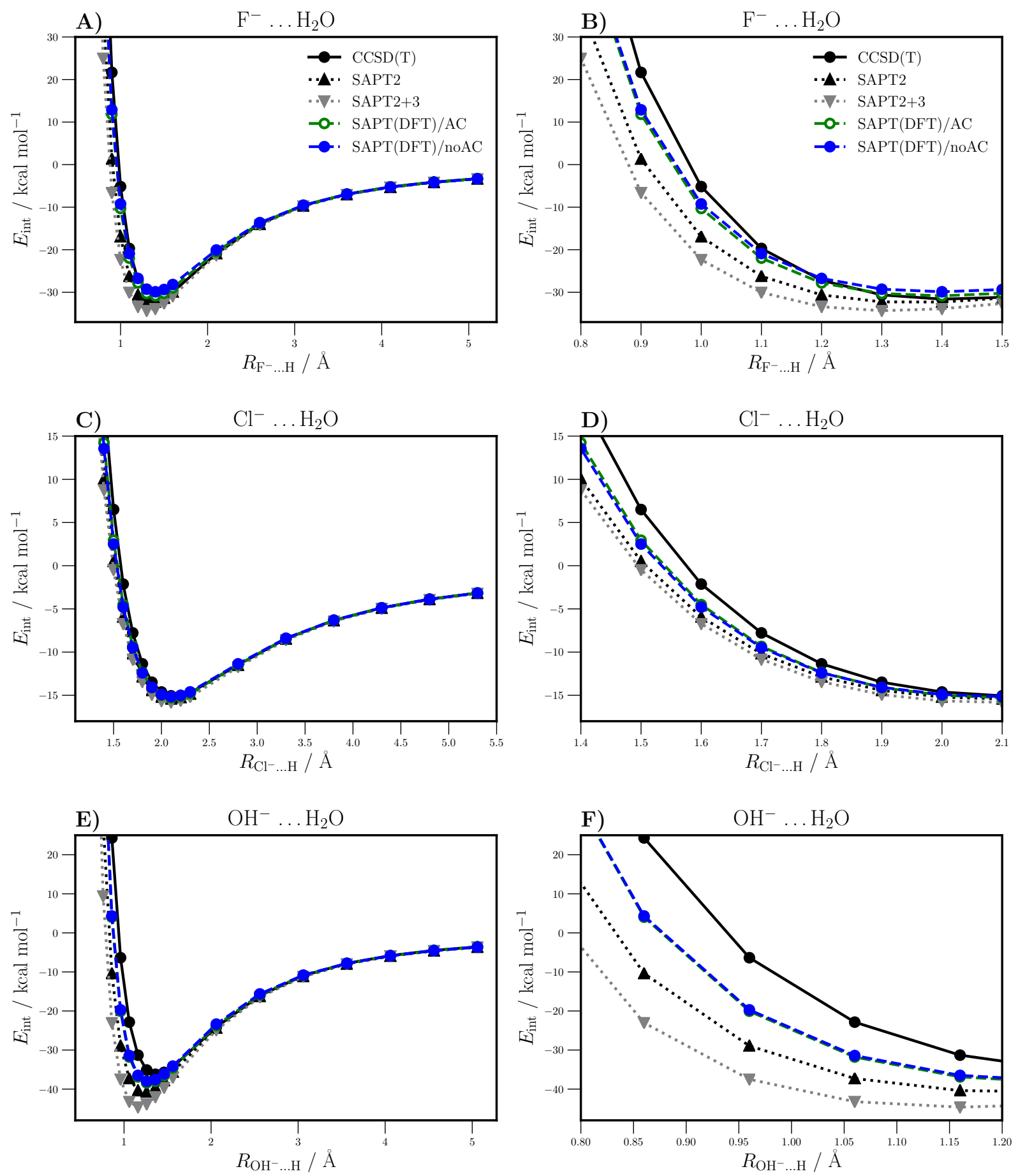

Figure S3: Influence of the Asymptotic Correction (AC) on anionic-water complexes ${ }^{1}$ in SAPT(DFT) using the asymptotically corrected PBE0 functional. First, the AC was applied on both anion and water molecule (AC). Then, the AC was kept on the water molecule but was removed for the anion (noAC). For SAPT(DFT) calculations, the $S^{2}$ approximation is only used for the $E_{\text {disp,exch }}^{(2)}$ energy. 
A comment on the use of the asymptotic-correction (AC) in SAPT(DFT): It is now wellknown that the $\mathrm{AC}$ is fundamental to the accuracy of the interaction energies and energy components in SAPT(DFT). Yet in the recent paper by Lao et al. ${ }^{1}$ it was recommended that the AC should not be used on anions as "The anionic systems were left without asymptotic correction since the XC potentials in these cases are short-ranged and do not decay as $1 / r . "$ We can demonstrate that this statement is incorrect and that not only does $v_{\mathrm{XC}} \rightarrow-1 / r$ for any finite molecular complex, but that the $\mathrm{AC}$ should always be used, at least in principle.

First of all, Lao et al. attribute the supposed short-range of the XC potential to Lee \& Burke $^{2}$ but this seems to be due to a misreading of this paper. Lee \& Burke correctly state that the Kohn-Sham potential, $v_{\mathrm{S}}$, for an anion is short-ranged, but they are clear that this is not the case for the $\mathrm{XC}$ potential $v_{\mathrm{XC}}$, which remains long-ranged, even for anions. This can be seen as follows: if an electron is pulled out of a species $X^{-}$, the interacting system is $X \cdots e^{-}$, which has no leading-order electrostatic interaction. Instead the interaction arises from a charge-polarization, which is relatively short-ranged and decays as $-1 / r^{4}$ (to leading order). If there are $N$ electrons in the anion with nuclear charge $Z$, then as we have $Z=N-1$, using $v_{\mathrm{s}}=v_{\mathrm{J}}+v_{\mathrm{ext}}+v_{\mathrm{XC}}$, and the long-range forms $v_{\mathrm{J}} \rightarrow+N / r$, and $v_{\mathrm{ext}} \rightarrow-Z / r$, it follows that (to leading order in $\left.1 / r\right) 0=+N / r-Z / r+v_{\mathrm{XC}}$, and so $v_{\mathrm{XC}} \rightarrow-1 / r$, as reported by Lee \& Burke.

Therefore the AC must be used for all species, including anions. However, the AC does not have much of an effect at very short distances from the nucleus as the switching function associated with most AC methods causes the $\mathrm{AC}$ to become appreciable only gradually, and at some distance from the valence region. This can be seen in the data presented in Table S1 which are graphed in Figure S3. Consequently, the AC has very little effect on the SAPT(DFT) energies involving the anions, where intermolecular separations are really very small. 


\section{Role of the $S^{2}$ approximation: Auxiliary data}

In Figure S4, Figure S5, Figure S6, and Figure S7 we present additional analysis of the significance of the $S^{2}$ approximation on the total interaction energy and on the first-order exchange and second-order exchange-induction energies. See the main paper for a discussion. 

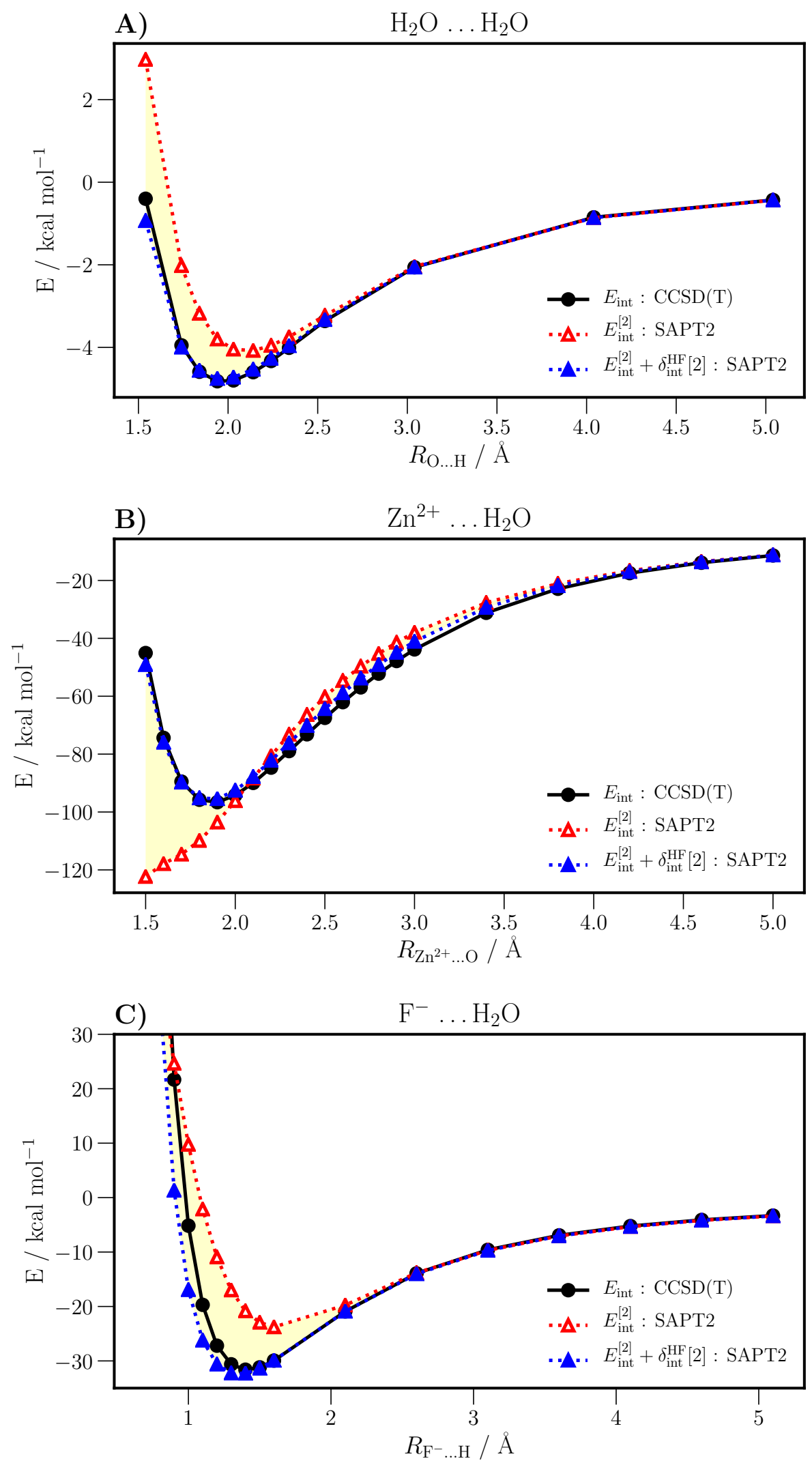

Figure S4: Contribution of the $\delta_{\text {int }}^{\mathrm{HF}}$ energy to the interaction energies from SAPT2. The $S^{2}$ approximation is used for the second-order exchange energies. The difference between the $E_{\text {int }}^{[2]}$ and $E_{\text {int }}^{[2]}+\delta_{\text {int }}^{\mathrm{HF}}[2]$ curves is the $\delta_{\text {int }}^{\mathrm{HF}}[2]$ contribution. This region is shaded yellow. 

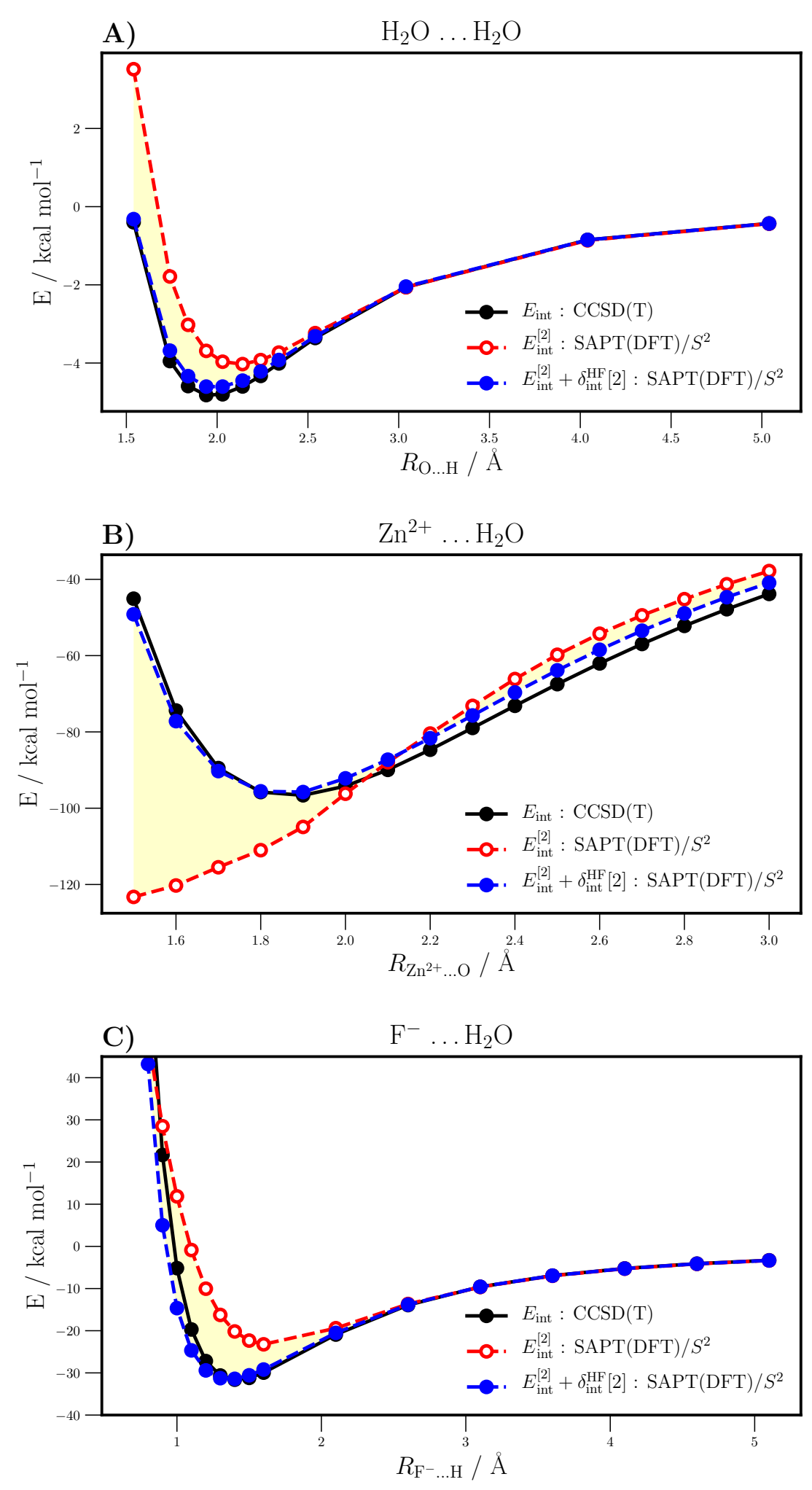

Figure S5: Contribution of the $\delta_{\text {int }}^{\mathrm{HF}}$ energy to the interaction energies from SAPT(DFT) using the asymptotically corrected PBE0 functional. The $S^{2}$ approximation is used only for the second-order exchange-induction energies. The difference between the $E_{\mathrm{int}}^{[2]}$ and $E_{\mathrm{int}}^{[2]}+\delta_{\mathrm{int}}^{\mathrm{HF}}[2]$ curves is the $\delta_{\text {int }}^{\mathrm{HF}}[2]$ contribution (shaded yellbw region). 

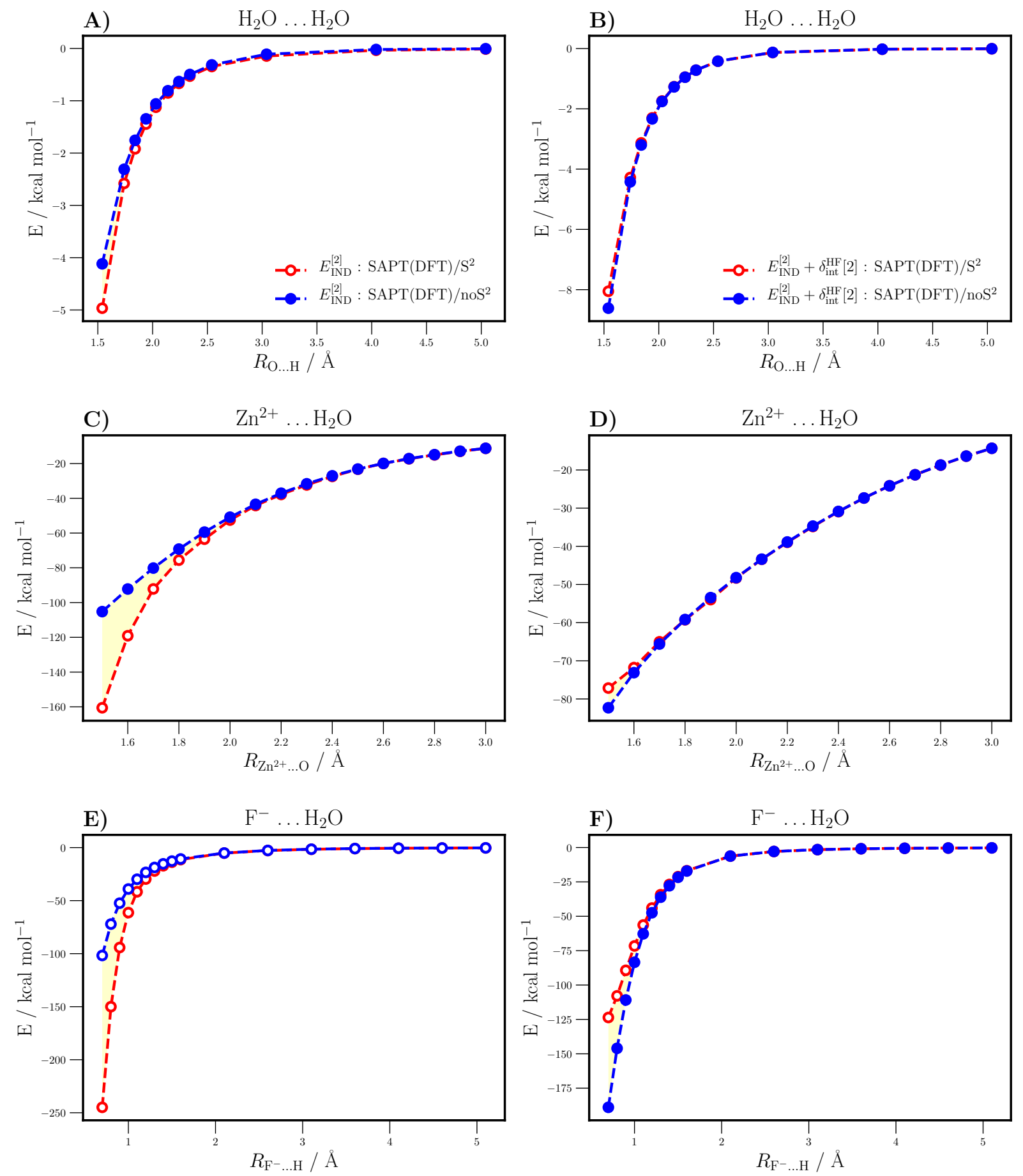

Figure S6: Contribution of the $\delta_{\text {int }}^{\text {HF }}$ energy to the total induction energies from SAPT(DFT) using the asymptotically corrected PBE0 functional. The $S^{2}$ approximation is used for the first and second-orders exchange energies. The difference between the SAPT(DFT) $/ S^{2}$ and $\mathrm{SAPT}(\mathrm{DFT}) /$ no $S^{2}$ curves is the effect of the $S^{2}$ (shaded yellow region). 

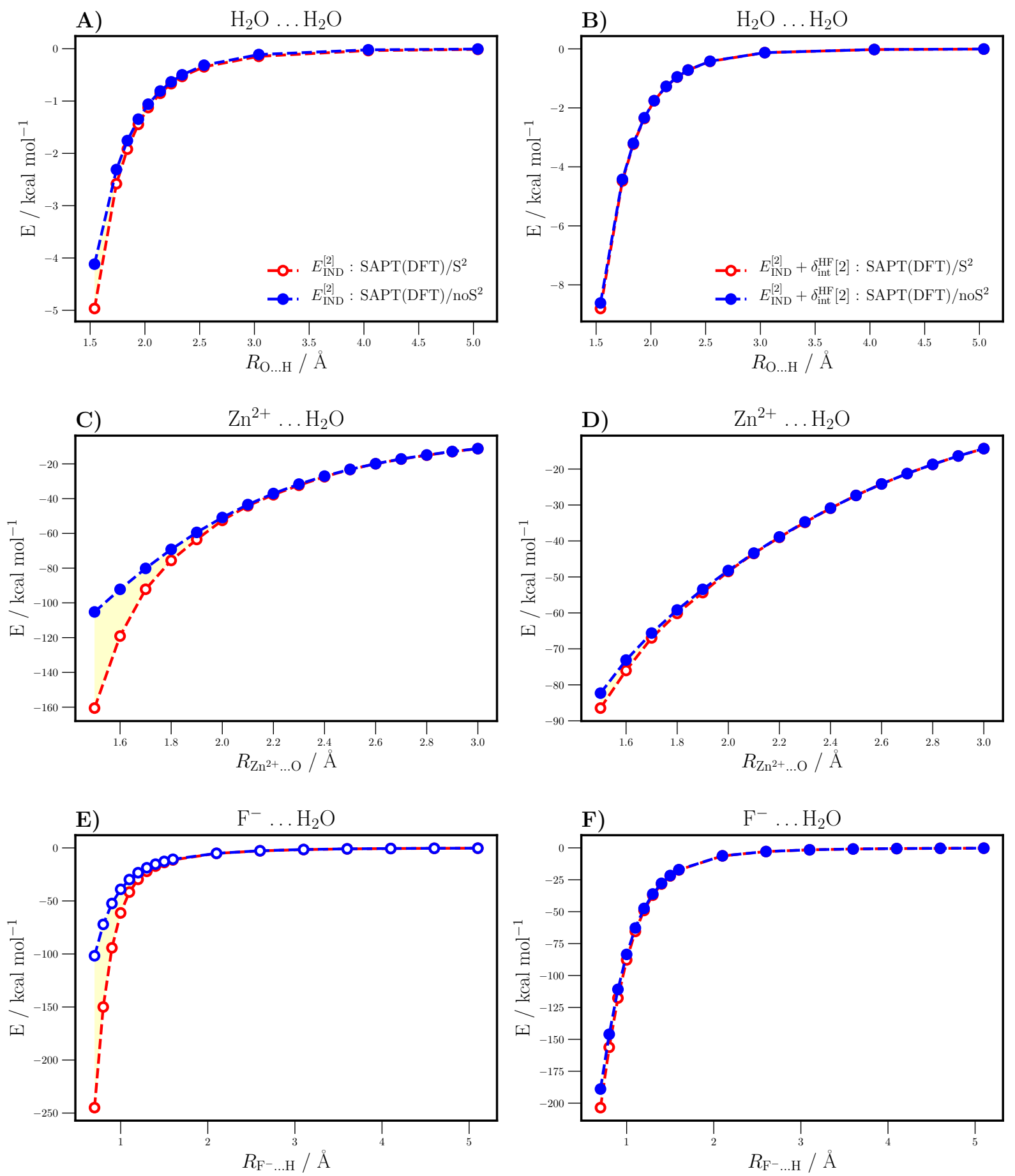

Figure S7: Contribution of the $\delta_{\text {int }}^{\mathrm{HF}}$ energy to the total induction energies from SAPT(DFT) using the asymptotically corrected PBE0 functional. The $S^{2}$ approximation is only used for the second-order exchange energies. The difference between the SAPT(DFT) $/ S^{2}$ and $\mathrm{SAPT}(\mathrm{DFT}) / \mathrm{no} S^{2}$ curves is the effect of the $S^{2}$ (shaded yellow region). 


\section{Separability of the interaction energy}

\subsection{Auxiliary plots}



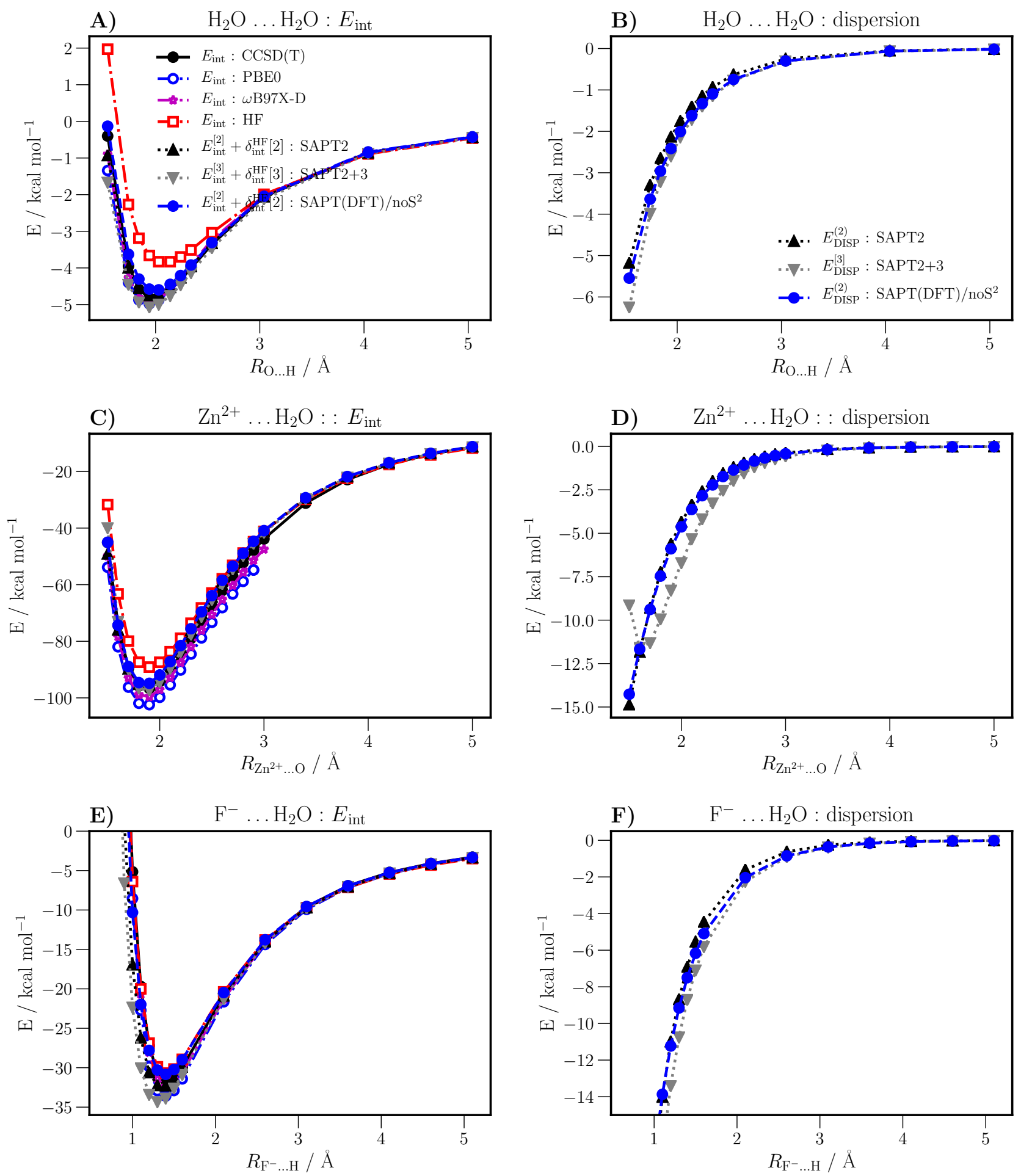

Figure S8: Comparison of the intermolecular interaction and dispersion contributions from SAPT models and SAPT(DFT) with those from supermolecular EDAs for the water dimer, $\mathrm{Zn}^{2+} \ldots \mathrm{H}_{2} \mathrm{O}$ and $\mathrm{F}^{-} \ldots \mathrm{H}_{2} \mathrm{O}$ complexes. The asymptotically corrected PBE0 functional is used for SAPT(DFT). In the SAPT models, the $S^{2}$ approximation is used for the second and third-order exchange energies. For SAPT(DFT) this approximation is present only in the $E_{\text {disp,exch }}^{(2)}$ energy. 


\subsection{Comparison of EDAs: ALMO, RVS \& CSOV}
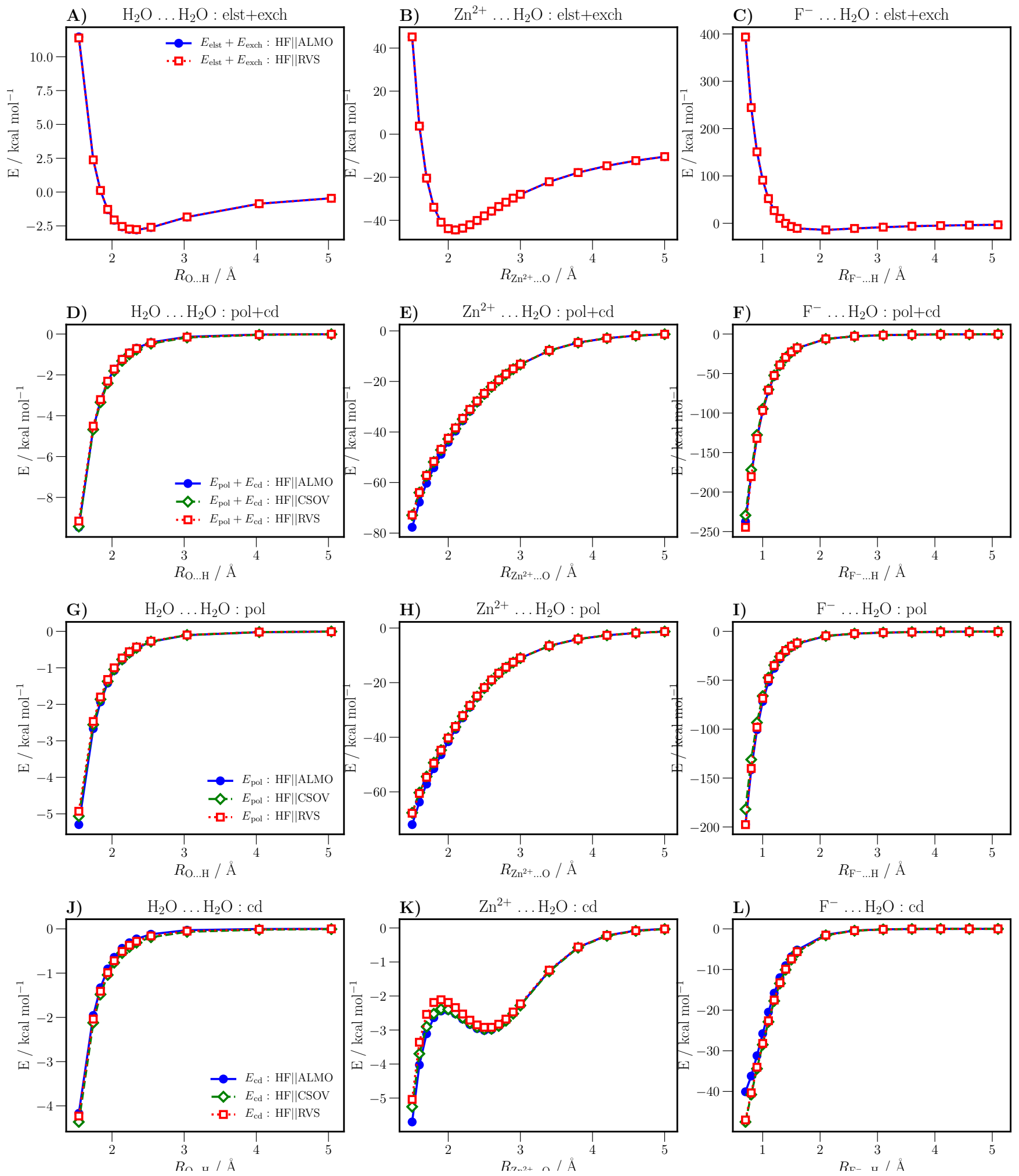

Figure S9: Comparison of energy components from the supermolecular EDAs based on HF wavefunctions. Results are displayed for the water dimer, $\mathrm{Zn}^{2+} \ldots \mathrm{H}_{2} \mathrm{O}$ complex and $\mathrm{F}^{-}$ ... $\mathrm{H}_{2} \mathrm{O}$ complexes. 
Here we provide a brief comparison of the ALMO EDA with two older EDAs: RVS and CSOV. Because of the way in which RVS has been implemented, it can only be used on Hartree-Fock wavefunctions, consequently we will make all comparisons using Hartree-Fock references. The main reason for this comparison is to get a sense of the effect of the different choices made in energy decomposition schemes on the energy components.

In Figure $\mathrm{S} 9$ we have displayed energies from these three EDAs for the $\left(\mathrm{H}_{2} \mathrm{O}\right)_{2}, \mathrm{Zn}^{2+}$ $\ldots \mathrm{H}_{2} \mathrm{O}$, and $\mathrm{F}^{-} \ldots \mathrm{H}_{2} \mathrm{O}$ complexes. Since the first-generation ALMO method does not decompose first-order energies into separate electrostatic and exchange-repulsion components, we have performed the comparisons on their sum: $E_{\text {elst }}+E_{\text {exch }}$. It is remarkable that all three methods give almost exactly the same $E_{\text {elst }}+E_{\text {exch }}$ energies for these systems, at all separations. Note that CSOV and RVS give rigorously identical $E_{\text {elst }}+E_{\text {exch }}$ energies so only those from RVS are shown. Before examining the individual $E_{\mathrm{POL}}$ and $E_{\mathrm{CD}}$ energies, we compare the EDAs on the sum $E_{\mathrm{POL}}+E_{\mathrm{CD}}$. Here we see small differences in the EDAs. For the $\left(\mathrm{H}_{2} \mathrm{O}\right)_{2}$ and $\mathrm{F}^{-} \ldots \mathrm{H}_{2} \mathrm{O}$ complexes the three EDAs yield essentially the same $E_{\mathrm{POL}}+E_{\mathrm{CD}}$ energies, but for $\mathrm{Zn}^{2+} \ldots \mathrm{H}_{2} \mathrm{O}$, while RVS and CSOV are nearly identical, ALMO results into somewhat larger (in magnitude) energies at short-range. However the differences in these methods are less than $10 \%$. This small difference in ALMO on one hand, and RVS and CSOV on the other, stems mostly from $E_{\mathrm{POL}}$. Note that RVS and CSOV are by no means identical as they yield different $E_{\mathrm{POL}}$ energies for the $\left(\mathrm{H}_{2} \mathrm{O}\right)_{2}$ and $\mathrm{F}^{-} \ldots \mathrm{H}_{2} \mathrm{O}$ complexes, but the differences between these methods are small. Note that $E_{\mathrm{POL}} \| \mathrm{CSOV}$ and $E_{\mathrm{POL}} \|$ RVS are the equivalent of "non-iterated" dipoles since the density of the polarizing monomer is frozen, and only the polarizable monomer is relaxed.

The largest relative differences between the three EDAs can be seen for the chargedelocalization energies, $E_{\mathrm{CD}}$, for the $\mathrm{Zn}^{2+} \ldots \mathrm{H}_{2} \mathrm{O}$ and $\mathrm{F}^{-} \ldots \mathrm{H}_{2} \mathrm{O}$ complexes. Though relatively large, these differences are numerically small. All EDAs show the same trends, even for the rather unusual case of $\mathrm{Zn}^{2+} \ldots \mathrm{H}_{2} \mathrm{O}$ where $E_{\mathrm{CD}}$ is seen to reach a minimum at just below $2.6 \AA$ before a maximum at around $1.9 \AA$. All three EDAs show this feature, which 
was reported in the first RVS analyses on $\mathrm{Zn}(\mathrm{II})$-ligand complexes by Garmer \& Gresh $^{3}$ and Gresh et al. ${ }^{4} \mathrm{~A}$ non-monotonous radial decay of $E_{\mathrm{CD}}$ was also found in the earliest RVS studies bearing on complexes of divalent cations with anionic ligands. ${ }^{5,6}$ It was more recently reported, with extended Gaussian basis sets, for actinide complexes ${ }^{7}$ where diabatization schemes were needed to prevent changes of electronic states upon deriving charge delocalization.

In summary, at least for these three systems, there are more similarities than differences in the ALMO, RVS and CSOV EDA schemes. This is perhaps encouraging as these schemes differ quite substantially in their implementation, but not, so it seems, in their physical content.

\section{Polarization models}

\section{$4.1 \quad\left(\mathrm{H}_{2} \mathrm{O}\right)_{2}$}

For the water dimer we have used the rank 3 polarization model from Gilmore et $a l .{ }^{8}$ This more uses anisotropic polarizability tensors that include terms up to rank three on all sites, and multipole moments up to rank 4 on all sites. Damping is done using the Tang-Toennies ${ }^{9}$ damping function with three damping coefficients for each of the site pair interactions (OO, $\mathrm{OH}$ and $\mathrm{HH}$ ). This model was developed using second-order regularised induction energies,

$E_{\text {IND }}^{(2)}($ Reg $)$, as described by Gilmore et al.. This model has been tested extensively on a wide range of water clusters.

Summary of polarization model data:

- Multipole moments: BS-ISA moments ${ }^{10}$ of rank 4 (L4mom) on all sites. PBE0/AC with daTZ basis.

- Polarizabilities: ISA-Pol localized polarizabilities ${ }^{11}$ of rank 3 (L3pol) on all sites. PBE0/AC with daTZ basis, and ALDA + CHF response kernel. 
- Damping: Tang-Toennies with the following damping parameters: $\beta_{\mathrm{pol}}^{\mathrm{OO}}=1.25$ a.u., $\beta_{\mathrm{pol}}^{\mathrm{OH}}=1.47$ a.u., and $\beta_{\mathrm{pol}}^{\mathrm{HH}}=2.0$ a.u.

All molecular properties were calculated with the CAMCASP ${ }^{12,13}$ program.

\section{$4.2 \mathrm{Zn}^{2+} \ldots \mathrm{H}_{2} \mathrm{O}$}

The polarization model for the $\mathrm{Zn}^{2+} \ldots \mathrm{H}_{2} \mathrm{O}$ system was developed using a similar methodology as for the $\left(\mathrm{H}_{2} \mathrm{O}\right)_{2}$. However here we found it necessary to use the Tang-Toennies damping functions with the SlaterFF form for $x$ (see equation (20) in the main text). Molecular properties (multipoles and polarizabilities) were computed using the BS-ISA ${ }^{10}$ and ISA-Pol ${ }^{11}$ algorithms using the PBE0/AC functional with the aug-cc-pVTZ basis. The GRAC asymptotic correction was used for water with shift of 0.13186 a.u. And for $\mathrm{Zn}^{2+}$ we used the same correction with shift 0.2201 a.u. All calculations were performed with the CAMCASP $\operatorname{program}^{12,13}$ with the DALTON2016 program used for the DFT calculations.

Summary of polarization model data:

- Multipole moments: BS-ISA moments of rank 4 (L4mom) on all sites. PBE0/AC with aTZ basis.

- Polarizabilities: ISA-Pol localized polarizabilities of rank 3 (L3pol) on all sites. PBE0/AC with aTZ basis, and ALDA+CHF response kernel.

- Damping: Tang-Toennies with the SlaterFF form of $x$ and one damping parameter for all site pairs: $\beta_{\mathrm{pol}}=1.59$ a.u.

The performance of this model may be gauged from Figure S10, from which we can see that the classical polarization model (at second order, that is, not iterated) fits the reference $E_{\mathrm{IND}}^{(2)}(\mathrm{Reg})$ regularized second-order induction energies from Reg-SAPT(DFT) reasonably well. Due to the small polarizability of $\mathrm{Zn}^{2+}$, the iterated polarization energy, $E_{\mathrm{POL}}^{(2-\infty)}$, does not differ from the second-order energies by much. 


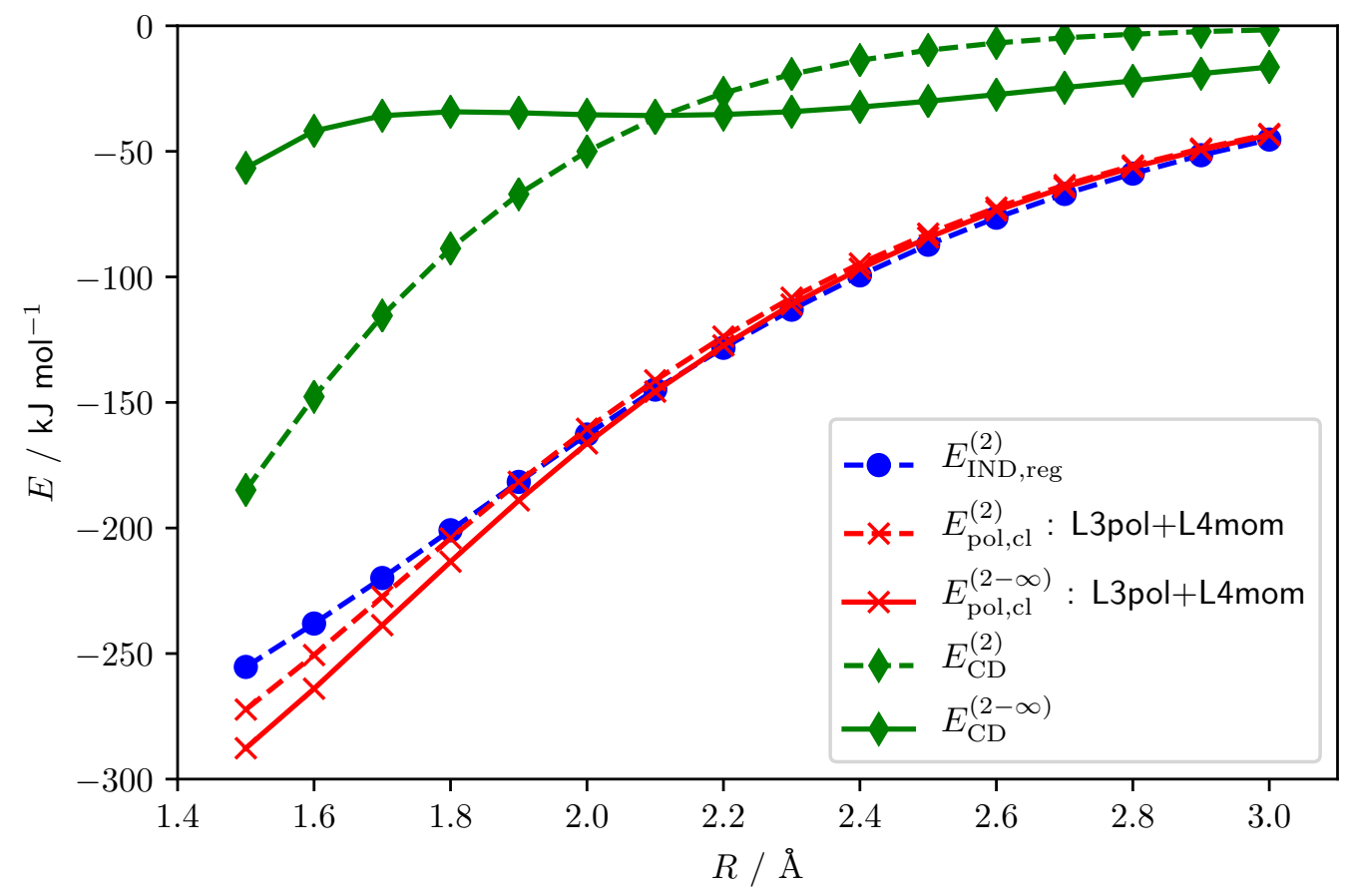

Figure S10: Polarization model for the $\mathrm{Zn}^{2+} \ldots \mathrm{H}_{2} \mathrm{O}$ complex. The damping for the classical polarization model energies $E_{\mathrm{pol}, \mathrm{cl}}^{(2)}$ was determined by fitting to the regularized secondorder induction energy $E_{\mathrm{IND}}^{(2)}(\mathrm{Reg})$. Also shown are the infinite-order polarization energies, $E_{\mathrm{pol}, \mathrm{cl}}^{(2-\infty)}$ obtained by iterating the classical polarization model to convergence, and the chargedelocalization energies at second and infinity order. The classical model energies are dependent on the polarization and electrostatic multipole models used; this is indicated using "L3pol+L4mom". 


\section{$4.3 \quad \mathrm{~F}^{-} \ldots \mathrm{H}_{2} \mathrm{O}$}

The polarization model for $\mathrm{F}^{-} \ldots \mathrm{H}_{2} \mathrm{O}$ was constructed using the following data:

- Multipole moments: BS-ISA moments of rank 4 (L4mom) on all sites. PBE0/AC with d-aug-cc-pVTZ basis on both $\mathrm{F}^{-}$and $\mathrm{H}_{2} \mathrm{O}$.

- Polarizabilities: ISA-Pol localized polarizabilities of rank 3 (L3pol) on all sites. PBE0/AC with daTZ basis, and ALDA+CHF response kernel. This basis is particularly important on the $\mathrm{F}^{-}$anion as it leads to a HOMO-LUMO gap commensurate with the gap in the aTZ/DC basis used in the SAPT(DFT) calculations. This leads to polarizabilities and dispersion coefficients (the latter not used in this paper) more consistent with the SAPT(DFT) energies.

- Damping: Tang-Toennies with the linear form of $x$ and one damping parameter for all site pairs: $\beta_{\mathrm{pol}}=1.25$ a.u.

In Figure S11 we display polarization and charge-delocalization energies for the $\mathrm{F}^{-} \ldots \mathrm{H}_{2} \mathrm{O}$ complex. These are presented as a function of the $\mathrm{F}^{-} \ldots \mathrm{H}$ separation. The classical polarization model results in a good fit to regularized second-order induction energy $E_{\text {IND }}^{(2)}(\operatorname{Reg})$. It is remarkable that the fit remains good even at the smallest separation of $0.68 \AA$. The infinite-order polarization, $E_{\mathrm{POL}}^{(2-\infty)}$, and charge-delocalization, $E_{\mathrm{CD}} 2-\infty$, energies are seen to be substantially larger (in magnitude) than the second-order counterparts.

Note that contrary to the $\mathrm{Zn}^{2+} \ldots \mathrm{H}_{2} \mathrm{O}$ system, here the iterated (infinite-order) energies are substantially different from the second-order energies. 


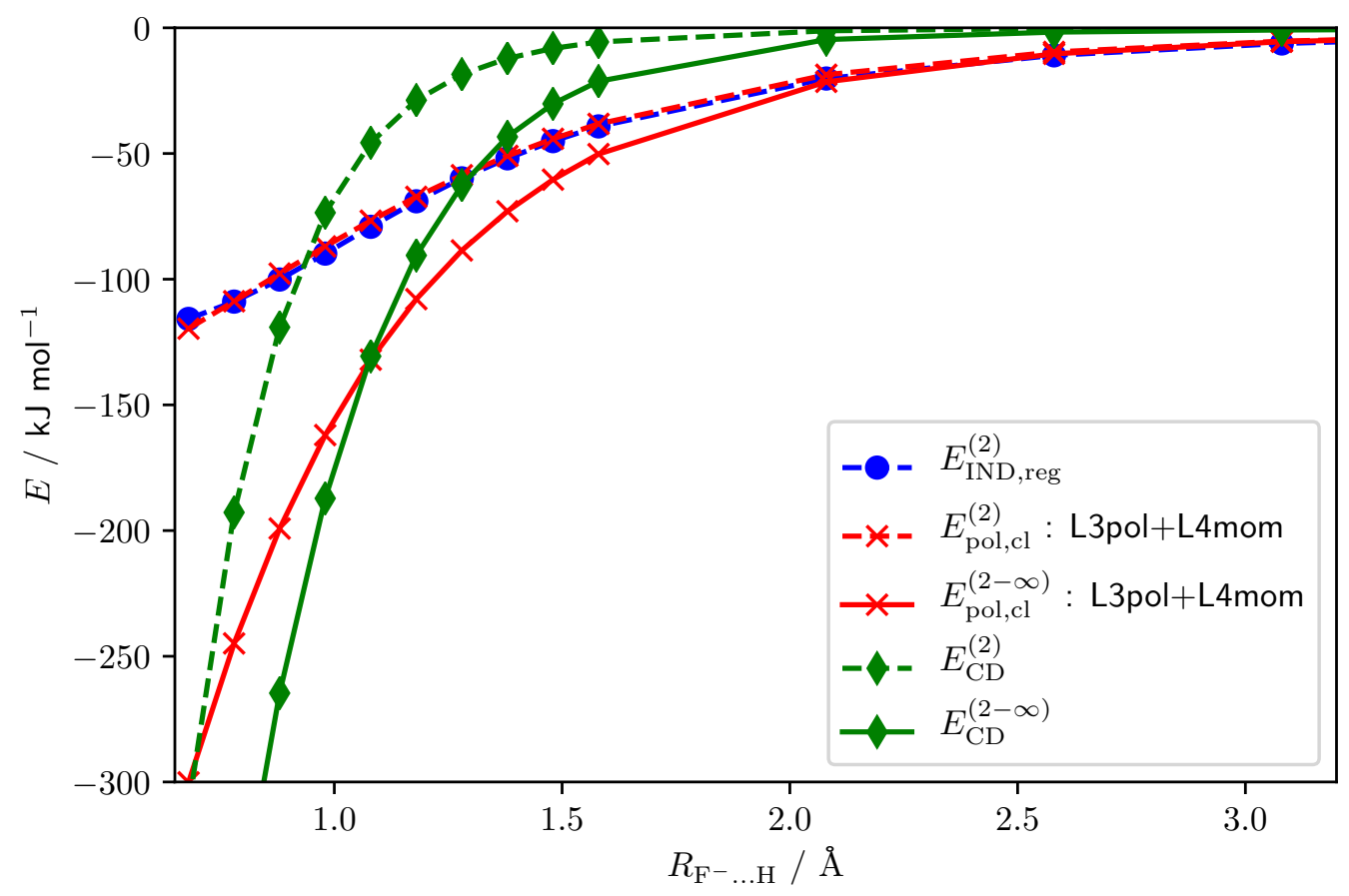

Figure S11: Polarization model for the $\mathrm{F}^{-} \ldots \mathrm{H}_{2} \mathrm{O}$ complex. The damping for the classical polarization model energies $E_{\mathrm{pol}, \mathrm{cl}}^{(2)}$ was determined by fitting to the regularized secondorder induction energy $E_{\mathrm{IND}}^{(2)}(\mathrm{Reg})$. Also shown are the infinite-order polarization energies, $E_{\mathrm{pol}, \mathrm{cl}}^{(2-\infty)}$ obtained by iterating the classical polarization model to convergence, and the chargedelocalization energies at second and infinity order. The classical model energies are dependent on the polarization and electrostatic multipole models used; this is indicated using "L3pol+L4mom". 


\section{Sensitivity of $E_{\text {elst }}^{(1)}+E_{\text {exch }}^{(1)}$ and $E_{\mathrm{CD}}$ to the wavefunction}
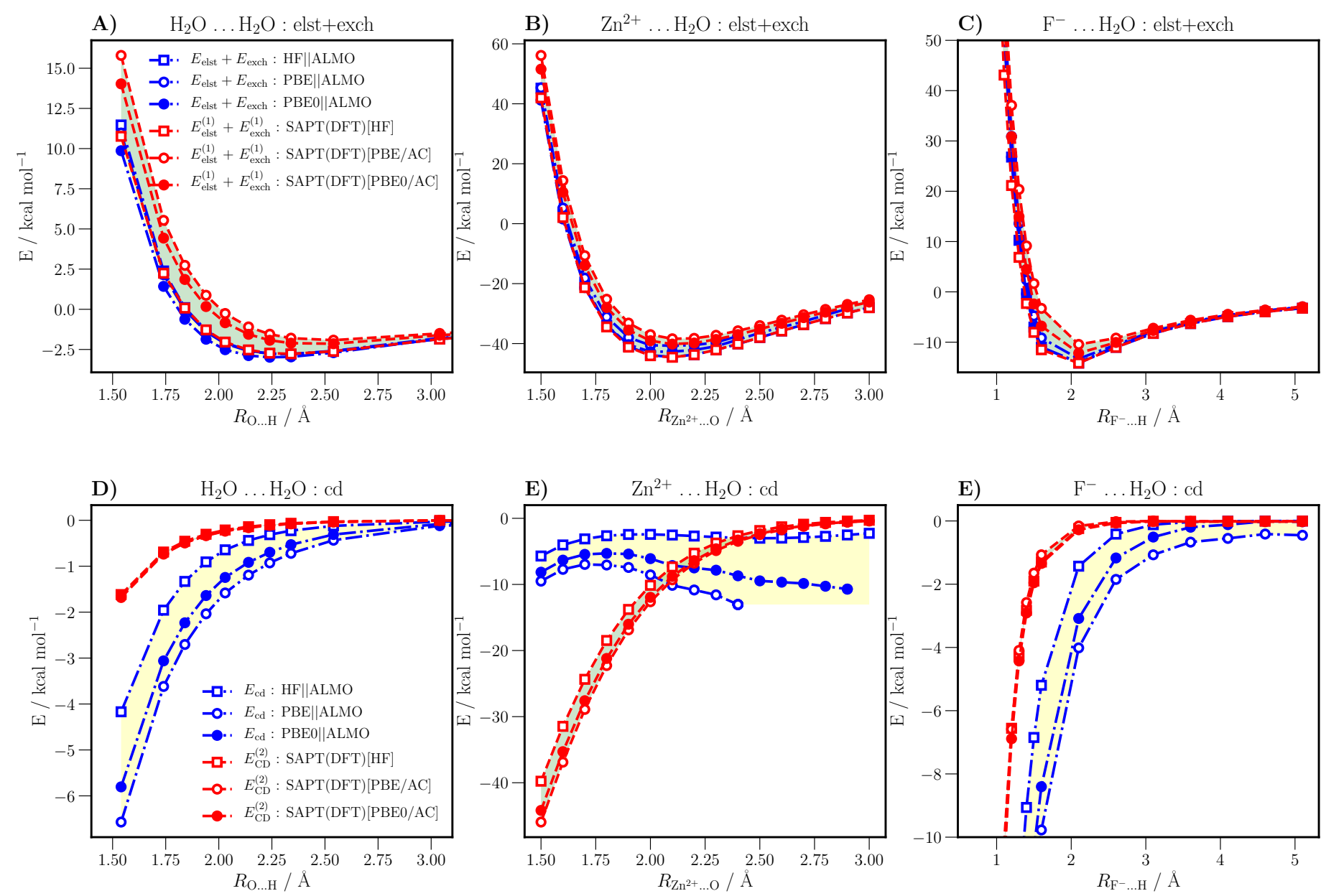

Figure S12: Sensitivity of the exchange and charge delocalization energies to the underlying electronic structure method for the water dimer, $\mathrm{Zn}^{2+} \ldots \mathrm{H}_{2} \mathrm{O}$ and $\mathrm{F}^{-} \ldots \mathrm{H}_{2} \mathrm{O}$ complexes. For SAPT(DFT) calculations, the $S^{2}$ approximation is only used for the $E_{\text {disp,exch }}^{(2)}$ energy.

Here we make a brief analysis of the sensitivity of energy components from SAPT(DFT) and ALMO to the underlying wavefunction. We fully expect some sensitivity to the underlying density and wavefunction: this is most easily seen in the asymptotic region, where, with the inclusion of correlation, we expect a less diffuse Hartree-Fock density to become more diffuse. Or, in the case of DFT, an overly diffuse PBE density will be expected to become 
less diffuse on inclusion of some fraction of exact exchange. These changes will give rise to changes in the higher ranking multipole moments, polarizabilities and the penetration and exchange energies. Additionally, we may expect the charge delocalization energy to vary. But how much of a change should we expect in going, say from Hartree-Fock to PBE0, through PBE?

In the context of polarizable force-field (polFF) development, some knowledge of the expected changes can be useful in deciding on weights and uncertainties. In Figure S12 we display, for the $\left(\mathrm{H}_{2} \mathrm{O}\right)_{2}, \mathrm{Zn}^{2+} \ldots \mathrm{H}_{2} \mathrm{O}$ and $\mathrm{F}^{-} \ldots \mathrm{H}_{2} \mathrm{O}$ complexes, $E_{\text {elst }}^{(1)}+E_{\text {exch }}^{(1)}$ and $E_{\mathrm{CD}}^{(2)}$ energies from SAPT(DFT) together with $E_{\text {elst }}+E_{\text {exch }}$ and $E_{\mathrm{CD}}$ energies from ALMO. To an extent, Hartree-Fock represents a worst-case scenario, so the difference between PBE and PBE0 results is what really matters. We see that for the sum of the electrostatic and exchange-repulsion energies, it is the supermolecular ALMO method that shows the smallest variability with the wavefunction. While SAPT(DFT) energies are more sensitive, much of the variation comes from the Hartree-Fock single-determinant wavefunction.

The SAPT(DFT) $E_{\text {elst }}^{(1)}+E_{\text {exch }}^{(1)}$ energies computed using the PBE and PBE0 orbitals are quite similar for all three complexes. This is reassuring as these two functionals would be the most commonly used functionals in the context of this method, and it is reassuring to see that the first-order energy sum is not too sensitive to which functional we use.

For the charge-delocalization energy we see a somewhat different pattern. Here it is the ALMO method that is more sensitive to the underlying wavefunction, while $E_{\mathrm{CD}}^{(2)}$ from $\mathrm{SAPT}(\mathrm{DFT})$ shows very little variation. If we focus only on the two density functionals, PBE and PBE0, we see a smaller variation of energies with ALMO, but these are still larger than the near insensitivity of $E_{\mathrm{CD}}^{(2)}$ from SAPT(DFT) to the use of PBE as opposed to PBE0. For ALMO, we see the largest variations in charge-delocalization energies for the $\mathrm{Zn}^{2+} \ldots \mathrm{H}_{2} \mathrm{O}$ complex: here both the PBE $\mid$ ALMO and PBE0||ALMO methods give $E_{\mathrm{CD}}$ energies that get unphysically larger with increasing separation, but HF||ALMO energies, though small, at least show a reasonable variation with separation. In contrast, as already explained in the 
main paper, the $E_{\mathrm{CD}}^{(2)}$ energies from $\mathrm{SAPT}(\mathrm{DFT})$ remain well-defined for this and the other complexes, at all separations.

We hesitate to draw any major conclusions from these observations, and merely point them out as indicative.

\section{Recommendations for SAPT(DFT) calculations}

We propose below a check-list of recommendations for consideration to derive SAPT(DFT) energies:

1. Remove the $S^{2}$ approximation from all interaction energies. Here we have not done so for the exchange-dispersion energy, but this is not expected to be a major approximation as this component is usually quite small. Removing this approximation leads to not only more accurate total interaction energies, but also results in each component being more physically meaningful without relying on error cancellations.

2. Underlying functional and kernel: While we have not made a systematic study of the dependency of SAPT(DFT) energies on the underlying functional, it is clear from this work and the work of the developers of this method, ${ }^{14-19}$ that asymptotically corrected PBE0 should be the functional of choice. In the SI to this paper, we have argued that the asymptotic correction should always be used, even for anions (in contrast to the recommendation made by a previous work ${ }^{1}$ ). We point out here that there is no unique way of performing the asymptotic correction and results will vary with the choice. ${ }^{14,20}$ Consequently when performing a SAPT(DFT) calculation, it is essential to be clear which correction is used and which shift was applied to the asymptotic region of the exchange-correlation potential. We recommend the GRAC asymptotic correction with shift computed as $\Delta=\epsilon_{\mathrm{HOMO}}+E_{I}$, where $\epsilon_{\mathrm{HOMO}}$ is the energy of the highest occupied molecular orbital, and $E_{I}$ is the (first) vertical ionisation energy (both in Hartree). We have used the hybrid ALDA+CHF kernel for all SAPT(DFT) 
calculations involving hybrid functionals. Note that because of the dependence of SAPT(DFT) on the functional, asymptotic correction, and kernel, there is no unique "SAPT(DFT)" interaction energy in the sense that SAPT2 or SAPT2+3 is uniquely specified. This means it is essential to be clear how the SAPT(DFT) calculation is performed: in general, the different choices in functional, asymptotic correction, ACshift, and kernel will make significant differences to the SAPT(DFT) energies.

3. Second-order polarization/charge-delocalization separation: We recommend splitting the second-order induction energy into these two components using Reg-SAPT(DFT) ${ }^{21}$ This method is available in the CAMCASP code, and has been demonstrated to yield accurate, predictive many-body models for water. ${ }^{8}$ One potential issue with this approach is the choice of the regularization parameter: we have used the value recommended value of $\eta=3.0$ a.u. from Misquitta, but there is evidence that this choice may not be suitable for all systems. This is currently under investigation in our groups.

4. Infinite-order polarization and charge-delocalization energies: Reg-SAPT(DFT) yields only the second-order polarization and charge-delocalization energies. To determine the infinite-order energies we follow the procedure described by Gilmore, Misquitta \& Stone ${ }^{8,13}$ and in this work. In brief, a classical polarization model is constructed by requiring that the $E_{\mathrm{IND}}^{(2)}(\mathrm{Reg})$ energies are reproduced with the non-iterated polarization model. On iteration to convergence the infinite-order polarization energy is determined, and using eq. (18), the infinite-order CD energy is determined. Details are provided in $§ 2.2 .1$. As the classical polarization model is an essential part of the force-field, this process does not add any new complexity to the procedure.

5. Terms of third- to infinite-order: The SAPT(DFT) interaction energies are defined to second-order using pure SAPT expressions. For polar systems terms of third- and higher-orders are essential (as has been demonstrated numerous times and can be seen from the data in Fig. 3, and these, largely higher-order induction contributions to 
the interaction energy can be approximated by the $\delta_{\text {int }}^{\mathrm{HF}}[2]$ correction. This term must be computed without the $S^{2}$ approximation to be matched with $\mathrm{SAPT}(\mathrm{DFT}) /$ no $S^{2}$ energies. We have not tackled the problem of higher-order terms for non-polar systems, or systems with mixed polar/non-polar interactions.

6. Basis sets: All the SAPT(DFT) calculations performed here have used the "MC+BS" (monomer-centred basis set with mid-bonds) basis type. ${ }^{22}$ Here the monomer orbitals and orbital energies used in SAPT(DFT) are calculated with a basis set containing functions on the monomer, as well as an $s$ - and $p$-function set on the "ghost" partner sites, and additionally a mid-bond set. These are termed the "far-bond" and "midbond" functions, and are needed to converge the charge-delocalization and dispersion energies. Note that there is no basis-set superposition error (BSSE) in the pure SAPT energies, so we have considerable latitude of freedom in constructing the basis sets. However, for the $\delta_{\text {int }}^{\mathrm{HF}}[2]$ energy calculation we need to use the "DCBS" (dimer-centred basis set) basis type in which the full dimer basis is used in all calculations and midbond functions are omitted. This is because the $\delta_{\mathrm{int}}^{\mathrm{HF}}[2]$ correction is a hybrid energy term with one part being the supermolecular Hartree-Fock interaction energy which is susceptible to the BSSE. So the counterpoise correction of Boys \& Bernardi is required. However mid-bond functions are not needed as there is no dispersion component to this energy term. Where possible, we recommend the aug-cc-pVTZ basis of Dunning for the main basis, and the $3 s 3 p 2 d 2 f$ mid-bond set from Podeszwa et al. ${ }^{23}$ together with the accompanying fitting basis from. These basis sets are supplied as part of the CAMCASP program in a format suitable for Psi4 and other codes. 


\section{$7 \quad$ Energy tables}

\section{$7.1 \quad\left(\mathrm{H}_{2} \mathrm{O}\right)_{2}$}

\begin{tabular}{rrrrr}
\hline \hline \multicolumn{5}{c}{$E_{\text {int }}$} \\
\hline $\mathrm{d}(\AA)$ & $\mathrm{CCSD}(\mathrm{T})$ & B3LYP & PBE0 & $\omega$ B97X-D \\
\hline 1.54 & -0.40 & -0.37 & -1.34 & -0.90 \\
1.74 & -3.95 & -3.76 & -4.39 & -4.26 \\
1.84 & -4.59 & -4.35 & -4.86 & -4.81 \\
1.94 & -4.82 & -4.54 & -4.97 & -4.96 \\
2.03 & -4.80 & -4.49 & -4.87 & -4.86 \\
2.14 & -4.60 & -4.28 & -4.61 & -4.59 \\
2.24 & -4.33 & -4.00 & -4.30 & -4.26 \\
2.34 & -4.01 & -3.69 & -3.97 & -3.91 \\
2.54 & -3.36 & -3.05 & -3.30 & -3.24 \\
3.04 & -2.06 & -1.84 & -2.02 & -2.02 \\
4.04 & -0.85 & -0.78 & -0.83 & -0.87 \\
5.04 & -0.43 & -0.41 & -0.42 & -0.44 \\
\hline \hline
\end{tabular}

\begin{tabular}{|c|c|c|c|c|c|c|c|c|}
\hline \multirow[b]{2}{*}{$\mathrm{d}(\AA)$} & \multicolumn{4}{|c|}{ PBE0||ALMO } & \multicolumn{4}{|c|}{$\omega B 97 X-D \| A L M O$} \\
\hline & $E_{\text {elst }}+E_{\text {exch }}$ & $E_{\mathrm{pol}}$ & $E_{\text {cd }}$ & $E_{\text {int }}$ & $E_{\text {elst }}+E_{\text {exch }}$ & $E_{\text {pol }}$ & $E_{\text {cd }}$ & $E_{\text {int }}$ \\
\hline 1.54 & 9.87 & -5.40 & -5.80 & -1.34 & 9.89 & -5.31 & -5.49 & -0.90 \\
\hline 1.74 & 1.43 & -2.76 & -3.06 & -4.40 & 1.41 & -2.80 & -2.87 & -4.26 \\
\hline 1.84 & -0.62 & -2.02 & -2.23 & -4.87 & -0.64 & -2.08 & -2.09 & -4.81 \\
\hline 1.94 & -1.86 & -1.48 & -1.64 & -4.98 & -1.87 & -1.56 & -1.53 & -4.96 \\
\hline 2.03 & -2.51 & -1.12 & -1.24 & -4.88 & -2.50 & -1.20 & -1.17 & -4.86 \\
\hline 2.14 & -2.88 & -0.82 & -0.92 & -4.62 & -2.85 & -0.89 & -0.85 & -4.59 \\
\hline 2.24 & -2.98 & -0.63 & -0.70 & -4.31 & -2.94 & -0.68 & -0.64 & -4.26 \\
\hline 2.34 & -2.96 & -0.48 & -0.53 & -3.97 & -2.91 & -0.52 & -0.49 & -3.91 \\
\hline 2.54 & -2.70 & -0.29 & -0.31 & -3.30 & -2.65 & -0.31 & -0.27 & -3.24 \\
\hline 3.04 & -1.83 & -0.11 & -0.08 & -2.02 & -1.85 & -0.11 & -0.06 & -2.02 \\
\hline 4.04 & -0.81 & -0.02 & -0.00 & -0.83 & -0.84 & -0.02 & -0.00 & -0.87 \\
\hline 5.04 & -0.41 & -0.01 & 0.00 & -0.42 & -0.43 & -0.01 & 0.00 & -0.44 \\
\hline
\end{tabular}




\begin{tabular}{rrrrrrrrrr}
\hline \hline & \multicolumn{3}{c}{ B3LYP ||CSOV } & & \multicolumn{5}{c}{ HF ||RVS } \\
\cline { 2 - 3 } \cline { 6 - 8 } $\mathrm{d}(\AA)$ & $E_{\text {pol }}$ & $E_{\text {cd }}$ & & $E_{\text {elst }}$ & $E_{\text {exch }}$ & $E_{\text {pol }}$ & $E_{\text {cd }}$ & $E_{\text {int }}$ \\
\hline 1.54 & -5.14 & -6.20 & & -19.11 & 30.50 & -4.93 & -4.23 & 1.97 \\
1.74 & -2.63 & -3.39 & & -12.44 & 14.82 & -2.47 & -2.04 & -2.27 \\
1.84 & -1.93 & -2.53 & & -10.20 & 10.32 & -1.80 & -1.41 & -3.19 \\
1.94 & -1.42 & -1.91 & & -8.45 & 7.16 & -1.32 & -0.99 & -3.66 \\
2.03 & -1.08 & -1.49 & & -7.17 & 5.12 & -1.00 & -0.72 & -3.83 \\
2.14 & -0.79 & -1.14 & & -6.00 & 3.46 & -0.73 & -0.51 & -3.83 \\
2.24 & -0.60 & -0.89 & & -5.14 & 2.41 & -0.56 & -0.37 & -3.70 \\
2.34 & -0.47 & -0.70 & & -4.44 & 1.67 & -0.43 & -0.28 & -3.51 \\
2.54 & -0.29 & -0.45 & & -3.41 & 0.81 & -0.27 & -0.16 & -3.04 \\
3.04 & -0.11 & -0.15 & & -1.97 & 0.13 & -0.10 & -0.05 & -1.99 \\
4.04 & -0.02 & -0.03 & & -0.86 & 0.00 & -0.02 & -0.01 & -0.89 \\
5.04 & -0.01 & -0.01 & & -0.46 & 0.00 & -0.01 & -0.00 & -0.46 \\
\hline \hline
\end{tabular}

\section{SAPT2}

\begin{tabular}{rrrrrrrrr}
\hline $\mathrm{d}(\AA)$ & $E_{\text {elst }}^{(1)}$ & $E_{\text {exch }}^{(1)}$ & $E_{\text {IND }}^{(2)}$ & $\delta_{\text {int }}^{\mathrm{HF}}[2]$ & $E_{\mathrm{DISP}}^{(2)}$ & $E_{\text {int }}^{[2]}$ & $E_{\text {int }}^{[2]}+\delta_{\text {int }}^{\mathrm{HF}}[2]$ & $E_{\mathrm{CD}}^{(2)}$ \\
\hline 1.54 & -19.31 & 32.93 & -5.47 & -3.90 & -5.18 & 2.97 & -0.93 & -2.28 \\
1.74 & -12.45 & 16.58 & -2.86 & -1.97 & -3.30 & -2.02 & -4.00 & -1.08 \\
1.84 & -10.14 & 11.73 & -2.12 & -1.38 & -2.65 & -3.18 & -4.56 & -0.76 \\
1.94 & -8.33 & 8.26 & -1.60 & -0.96 & -2.13 & -3.80 & -4.76 & -0.55 \\
2.03 & -7.02 & 5.97 & -1.24 & -0.68 & -1.75 & -4.05 & -4.73 & -0.41 \\
2.14 & -5.82 & 4.08 & -0.93 & -0.46 & -1.40 & -4.08 & -4.53 & -0.29 \\
2.24 & -4.96 & 2.87 & -0.73 & -0.31 & -1.14 & -3.95 & -4.27 & -0.21 \\
2.34 & -4.25 & 2.01 & -0.57 & -0.22 & -0.93 & -3.75 & -3.96 & -0.16 \\
2.54 & -3.23 & 0.98 & -0.35 & -0.10 & -0.63 & -3.23 & -3.33 & -0.09 \\
3.04 & -1.84 & 0.16 & -0.12 & -0.02 & -0.25 & -2.04 & -2.06 & -0.02 \\
4.04 & -0.79 & 0.00 & -0.02 & -0.00 & -0.05 & -0.86 & -0.86 & -0.00 \\
5.04 & -0.41 & 0.00 & -0.01 & -0.00 & -0.02 & -0.43 & -0.44 & -0.00 \\
\hline \hline
\end{tabular}




\begin{tabular}{rrrrrrrrrr}
\hline \hline \multicolumn{10}{c}{ SAPT2 +3} \\
\hline $\mathrm{d}(\AA)$ & $E_{\text {elst }}^{(1)}$ & $E_{\text {exch }}^{(1)}$ & $E_{\text {IND }}^{[3]}$ & $\delta_{\text {int }}^{\mathrm{HF}}[3]$ & $E_{\text {DISP }}^{[3]}$ & $E_{\text {int }}^{[3]}$ & $E_{\text {int }}^{[3]}+\delta_{\text {int }}^{\mathrm{HF}}[3]$ & $E_{\mathrm{CD}}^{[3]}$ \\
\hline 1.54 & -18.98 & 32.93 & -7.74 & -1.64 & -6.25 & -0.03 & -1.67 & -4.15 \\
1.74 & -12.22 & 16.58 & -3.69 & -1.14 & -3.99 & -3.32 & -4.46 & -1.73 \\
1.84 & -9.95 & 11.73 & -2.66 & -0.85 & -3.22 & -4.09 & -4.94 & -1.15 \\
1.94 & -8.18 & 8.26 & -1.94 & -0.61 & -2.60 & -4.46 & -5.07 & -0.78 \\
2.03 & -6.91 & 5.97 & -1.48 & -0.44 & -2.14 & -4.56 & -5.00 & -0.55 \\
2.14 & -5.74 & 4.08 & -1.09 & -0.30 & -1.71 & -4.46 & -4.76 & -0.38 \\
2.24 & -4.90 & 2.87 & -0.83 & -0.21 & -1.40 & -4.26 & -4.47 & -0.27 \\
2.34 & -4.21 & 2.01 & -0.64 & -0.14 & -1.14 & -3.99 & -4.13 & -0.20 \\
2.54 & -3.21 & 0.98 & -0.39 & -0.07 & -0.77 & -3.39 & -3.46 & -0.10 \\
3.04 & -1.84 & 0.16 & -0.13 & -0.01 & -0.30 & -2.11 & -2.12 & -0.02 \\
4.04 & -0.80 & 0.00 & -0.02 & -0.00 & -0.06 & -0.88 & -0.88 & -0.00 \\
5.04 & -0.42 & 0.00 & -0.01 & -0.00 & -0.02 & -0.44 & -0.44 & -0.00 \\
\hline \hline
\end{tabular}

\begin{tabular}{ccccccccccc}
\hline \hline \multicolumn{10}{c}{$\mathrm{SAPT}(\mathrm{DFT})\left[\mathrm{PBE} 0 / \mathrm{AC} / S^{2}\right]$} \\
\hline $\mathrm{d}(\AA)$ & $E_{\text {elst }}^{(1)}$ & $E_{\text {exch }}^{(1)}$ & $E_{\text {IND }}^{(2)}$ & $\delta_{\text {int }}^{\mathrm{HF}}[2]$ & $E_{\text {DISP }}^{(2)}$ & $E_{\text {int }}^{[2]}$ & $E_{\text {int }}^{[2]}+\delta_{\text {int }}^{\mathrm{HF}}[2]$ & $E_{\mathrm{CD}}^{(2)}$ & $E_{\mathrm{POL}}^{(2)}$ \\
\hline 1.54 & -19.05 & 32.20 & -4.97 & -3.09 & -5.55 & 2.63 & -0.45 & -2.36 & -2.60 \\
1.74 & -12.19 & 16.37 & -2.58 & -1.70 & -3.64 & -2.03 & -3.73 & -0.95 & -1.63 \\
1.84 & -9.89 & 11.62 & -1.92 & -1.21 & -2.96 & -3.15 & -4.36 & -0.63 & -1.29 \\
1.94 & -8.11 & 8.22 & -1.45 & -0.86 & -2.41 & -3.76 & -4.61 & -0.42 & -1.03 \\
2.03 & -6.83 & 5.96 & -1.12 & -0.62 & -2.01 & -4.00 & -4.62 & -0.29 & -0.83 \\
2.14 & -5.66 & 4.08 & -0.85 & -0.41 & -1.62 & -4.05 & -4.46 & -0.20 & -0.66 \\
2.24 & -4.80 & 2.87 & -0.67 & -0.28 & -1.33 & -3.93 & -4.21 & -0.14 & -0.53 \\
2.34 & -4.12 & 2.01 & -0.53 & -0.19 & -1.10 & -3.74 & -3.93 & -0.11 & -0.42 \\
2.54 & -3.13 & 0.99 & -0.35 & -0.08 & -0.75 & -3.24 & -3.31 & -0.07 & -0.28 \\
3.04 & -1.78 & 0.16 & -0.15 & 0.01 & -0.30 & -2.06 & -2.05 & -0.04 & -0.11 \\
4.04 & -0.77 & 0.00 & -0.03 & 0.01 & -0.06 & -0.86 & -0.85 & -0.02 & -0.02 \\
5.04 & -0.41 & 0.00 & -0.01 & 0.01 & -0.02 & -0.44 & -0.43 & -0.01 & -0.01 \\
\hline \hline
\end{tabular}




\begin{tabular}{rrrrrrrrrrrr}
\hline \hline \multicolumn{10}{c}{$\mathrm{SAPT}(\mathrm{DFT})\left[\mathrm{PBE} / \mathrm{AC} / \mathrm{no} S^{2}\right]$} \\
\hline $\mathrm{d}(\AA)$ & $E_{\text {elst }}^{(1)}$ & $E_{\text {exch }}^{(1)}$ & $E_{\mathrm{IND}}^{(2)}$ & $\delta_{\text {int }}^{\mathrm{HF}}[2]$ & $E_{\mathrm{DISP}}^{(2)}$ & $E_{\text {int }}^{[2]}$ & $E_{\text {int }}^{[2]}+\delta_{\text {int }}^{\mathrm{HF}}[2]$ & $E_{\mathrm{CD}}^{(2)}$ & $E_{\mathrm{POL}}^{(2)}$ & $E_{\mathrm{CD}}^{(2-\infty)}$ & $E_{\mathrm{POL}}^{(2-\infty)}$ \\
\hline 1.54 & -19.05 & 33.08 & -4.12 & -4.50 & -5.55 & 4.36 & -0.13 & -1.64 & -2.47 & -4.81 & -3.80 \\
1.74 & -12.19 & 16.62 & -2.31 & -2.11 & -3.64 & -1.52 & -3.63 & -0.72 & -1.59 & -2.26 & -2.16 \\
1.84 & -9.89 & 11.75 & -1.76 & -1.44 & -2.96 & -2.86 & -4.30 & -0.48 & -1.27 & -1.55 & -1.65 \\
1.94 & -8.11 & 8.29 & -1.35 & -0.99 & -2.41 & -3.59 & -4.58 & -0.33 & -1.02 & -1.07 & -1.27 \\
2.03 & -6.83 & 5.99 & -1.06 & -0.70 & -2.01 & -3.90 & -4.60 & -0.23 & -0.83 & -0.76 & -1.00 \\
2.14 & -5.66 & 4.10 & -0.81 & -0.46 & -1.62 & -3.98 & -4.45 & -0.16 & -0.65 & -0.51 & -0.77 \\
2.24 & -4.80 & 2.88 & -0.63 & -0.32 & -1.33 & -3.89 & -4.21 & -0.11 & -0.52 & -0.35 & -0.60 \\
2.34 & -4.12 & 2.02 & -0.50 & -0.22 & -1.10 & -3.70 & -3.92 & -0.08 & -0.42 & -0.24 & -0.48 \\
2.54 & -3.13 & 0.99 & -0.31 & -0.10 & -0.75 & -3.20 & -3.31 & -0.03 & -0.28 & -0.12 & -0.30 \\
3.04 & -1.78 & 0.16 & -0.11 & -0.02 & -0.30 & -2.03 & -2.04 & -0.00 & -0.11 & -0.02 & -0.11 \\
4.04 & -0.77 & 0.00 & -0.02 & -0.00 & -0.06 & -0.85 & -0.85 & -0.00 & -0.02 & 0.00 & -0.02 \\
5.04 & -0.41 & 0.00 & -0.01 & -0.00 & -0.02 & -0.43 & -0.43 & -0.00 & -0.01 & 0.00 & -0.01 \\
\hline \hline
\end{tabular}

$7.2 \quad \mathrm{Zn}^{2+} \ldots \mathrm{H}_{2} \mathrm{O}$

\begin{tabular}{crrrr}
\hline \hline \multicolumn{5}{c}{$E_{\text {int }}$} \\
\hline $\mathrm{d}(\AA)$ & $\mathrm{CCSD}(\mathrm{T})$ & B3LYP & PBE0 & $\omega$ B97X-D \\
\hline 1.5 & -45.08 & -53.06 & -53.69 & -49.79 \\
1.6 & -74.38 & -81.73 & -81.79 & -78.21 \\
1.7 & -89.49 & -96.49 & -96.07 & -92.78 \\
1.8 & -95.77 & -102.62 & -101.79 & -98.77 \\
1.9 & -96.62 & -103.47 & -102.30 & -99.42 \\
2.0 & -94.22 & -101.16 & -99.70 & -97.01 \\
2.1 & -89.95 & -97.05 & -95.34 & -92.71 \\
2.2 & -84.67 & -92.00 & -90.07 & -87.50 \\
2.3 & -78.96 & -86.55 & -84.43 & -81.85 \\
2.4 & -73.16 & -81.06 & -78.76 & -76.12 \\
2.5 & -67.48 & -75.73 & -73.27 & -70.56 \\
2.6 & -62.05 & -70.70 & -68.07 & -65.24 \\
2.7 & -56.95 & -66.03 & -63.24 & -60.25 \\
2.8 & -52.21 & -61.76 & -58.81 & -55.63 \\
2.9 & -47.84 & -57.92 & -54.79 & -51.38 \\
3.0 & -43.83 & -56.49 & -51.17 & -47.49 \\
3.4 & -31.19 & -52.56 & not conv. & -35.29 \\
3.8 & -22.88 & -52.82 & -45.78 & -27.40 \\
4.2 & -17.47 & -54.50 & -46.78 & -22.58 \\
4.6 & -13.88 & -56.58 & -48.37 & -19.76 \\
5.0 & -11.38 & -58.70 & -50.12 & -31.66 \\
\hline \hline & & & & \\
\hline
\end{tabular}




\begin{tabular}{|c|c|c|c|c|c|c|c|c|c|}
\hline \multirow[b]{2}{*}{$\mathrm{d}(\AA)$} & \multicolumn{4}{|c|}{ PBE0||ALMO } & \multicolumn{5}{|c|}{$\omega \mathrm{B} 97 \mathrm{X}-\mathrm{D} \| \mathrm{ALMO}$} \\
\hline & $E_{\text {elst }}+E_{\text {exch }}$ & $E_{\mathrm{pol}}$ & $E_{\mathrm{cd}}$ & $E_{\text {int }}$ & $E_{\text {elst }}$ & $+E_{\text {exch }}$ & $E_{\mathrm{pol}}$ & $E_{\mathrm{cd}}$ & $E_{\text {int }}$ \\
\hline 1.5 & 41.20 & -86.90 & -8.15 & -53.85 & & 44.28 & -86.48 & -7.72 & -49.92 \\
\hline 1.6 & 1.56 & -77.18 & -6.33 & -81.95 & & 4.15 & -76.64 & -5.91 & -78.40 \\
\hline 1.7 & -21.33 & -69.42 & -5.45 & -96.21 & & -19.15 & -68.78 & -5.03 & -92.96 \\
\hline 1.8 & -33.89 & -62.72 & -5.30 & -101.91 & & -32.07 & -62.00 & -4.84 & -98.91 \\
\hline 1.9 & -40.35 & -56.65 & -5.40 & -102.41 & & -38.64 & -55.88 & -5.08 & -99.60 \\
\hline 2.0 & -42.71 & -50.99 & -6.10 & -99.79 & & -41.41 & -50.13 & -5.55 & -97.09 \\
\hline 2.1 & -42.69 & -45.59 & -7.15 & -95.42 & & -41.92 & -44.74 & -6.16 & -92.82 \\
\hline 2.2 & -42.15 & -40.51 & -7.50 & -90.16 & & -41.14 & -39.66 & -6.77 & -87.58 \\
\hline 2.3 & -40.89 & -35.78 & -7.85 & -84.52 & & -39.62 & -34.93 & -7.36 & -81.90 \\
\hline 2.4 & -38.72 & -31.40 & -8.70 & -78.82 & & -37.74 & -30.59 & -7.86 & -76.19 \\
\hline 2.5 & -36.42 & -27.43 & -9.46 & -73.31 & & -35.72 & -26.68 & -8.20 & -70.60 \\
\hline 2.6 & -34.55 & -23.90 & -9.66 & -68.11 & & -33.65 & -23.20 & -8.43 & -65.28 \\
\hline 2.7 & -32.62 & -20.79 & -9.86 & -63.27 & & -31.64 & -20.14 & -8.51 & -60.29 \\
\hline 2.8 & -30.48 & -18.07 & -10.27 & -58.83 & & -29.82 & -17.46 & -8.38 & -55.66 \\
\hline 2.9 & -28.39 & -15.70 & -10.71 & -54.80 & & -28.20 & -15.17 & -8.04 & -51.40 \\
\hline 3.0 & \multicolumn{4}{|c|}{ not converged } & & -26.71 & -13.18 & -7.62 & -47.51 \\
\hline 3.4 & \multicolumn{4}{|c|}{$"$} & & -21.44 & -7.74 & -6.15 & -35.33 \\
\hline 3.8 & \multicolumn{4}{|c|}{$\|$} & & -17.30 & -4.78 & -5.39 & -27.47 \\
\hline 4.2 & \multicolumn{4}{|c|}{$"$} & \multicolumn{5}{|c|}{ not converged } \\
\hline 4.6 & \multicolumn{4}{|c|}{ 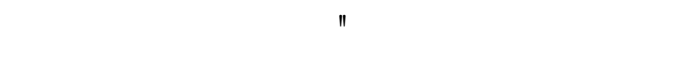 } & \multicolumn{5}{|c|}{$"$} \\
\hline 5.0 & \multicolumn{4}{|c|}{$"$} & \multicolumn{5}{|c|}{$"$} \\
\hline
\end{tabular}




\begin{tabular}{crrrrrrrr}
\hline \hline & \multicolumn{3}{c}{ B3LYP||CSOV } & & \multicolumn{5}{c}{ HF||RVS } \\
\cline { 2 - 3 } \cline { 6 - 8 } $\mathrm{d}(\AA)$ & $E_{\text {pol }}$ & $E_{\text {cd }}$ & & $E_{\text {elst }}$ & $E_{\text {exch }}$ & $E_{\text {pol }}$ & $E_{\text {cd }}$ & $E_{\text {int }}$ \\
\hline 1.5 & -81.43 & -8.13 & & -193.71 & 238.90 & -67.82 & -5.04 & -31.69 \\
1.6 & -72.93 & -6.42 & & -150.72 & 154.46 & -60.57 & -3.36 & -63.25 \\
1.7 & -66.30 & -5.72 & & -120.52 & 100.10 & -54.66 & -2.54 & -79.95 \\
1.8 & -60.23 & -5.59 & & -98.88 & 64.95 & -49.49 & -2.19 & -87.39 \\
1.9 & -54.89 & -5.89 & & -83.05 & 42.15 & -44.75 & -2.11 & -89.12 \\
2.0 & -50.14 & -6.53 & & -71.22 & 27.33 & -40.29 & -2.19 & -87.41 \\
2.1 & -45.23 & -7.32 & & -62.18 & 17.70 & -36.07 & -2.34 & -83.69 \\
2.2 & -40.30 & -8.19 & & -55.12 & 11.45 & -32.08 & -2.53 & -78.91 \\
2.3 & -35.87 & -9.15 & & -49.47 & 7.39 & -28.35 & -2.71 & -73.63 \\
2.4 & -31.93 & -10.15 & & -44.86 & 4.77 & -24.91 & -2.85 & -68.23 \\
2.5 & -28.14 & -11.05 & & -41.02 & 3.08 & -21.79 & -2.92 & -62.94 \\
2.6 & -24.46 & -11.79 & & -37.75 & 2.00 & -19.00 & -2.92 & -57.90 \\
2.7 & -21.20 & -12.38 & & -34.92 & 1.30 & -16.54 & -2.83 & -53.17 \\
2.8 & -18.55 & -12.87 & & -32.45 & 0.85 & -14.39 & -2.68 & -48.81 \\
2.9 & -16.32 & -13.34 & & -30.25 & 0.55 & -12.53 & -2.47 & -44.81 \\
3.0 & -14.28 & -13.84 & & -28.29 & 0.37 & -10.93 & -2.23 & -41.17 \\
3.4 & -8.20 & -15.35 & & -22.15 & 0.07 & -6.49 & -1.24 & -29.84 \\
3.8 & -4.94 & -0.07 & & -17.84 & 0.02 & -4.04 & -0.56 & -22.44 \\
4.2 & -3.20 & -0.36 & & -14.67 & 0.00 & -2.65 & -0.22 & -17.54 \\
4.6 & -2.13 & -0.54 & & -12.27 & 0.00 & -1.81 & -0.08 & -14.17 \\
5.0 & -1.48 & -0.11 & & -10.42 & 0.00 & -1.29 & -0.03 & -11.73 \\
\hline \hline
\end{tabular}




\begin{tabular}{rrrrrrrrr}
\hline \hline & \multicolumn{7}{c}{ SAPT2 } \\
\hline $\mathrm{d}(\AA)$ & $E_{\text {elst }}^{(1)}$ & $E_{\text {exch }}^{(1)}$ & $E_{\text {IND }}^{(2)}$ & $\delta_{\text {int }}^{\text {HF }}[2]$ & $E_{\text {DISP }}^{(2)}$ & $E_{\text {int }}^{[2]}$ & $E_{\text {int }}^{[2]}+\delta_{\text {int }}^{\text {HF }}[2]$ & $E_{\text {CD }}^{(2)}$ \\
\hline 1.5 & -193.28 & 245.14 & -159.33 & 73.19 & -14.87 & -122.34 & -49.14 & -14.20 \\
1.6 & -149.59 & 161.03 & -117.51 & 41.93 & -11.84 & -117.91 & -75.98 & -3.96 \\
1.7 & -118.84 & 105.90 & -92.38 & 24.89 & -9.30 & -114.63 & -89.74 & -0.32 \\
1.8 & -96.86 & 69.71 & -75.50 & 14.71 & -7.25 & -109.90 & -95.19 & 0.42 \\
1.9 & -80.87 & 45.94 & -63.00 & 8.11 & -5.62 & -103.55 & -95.44 & 0.07 \\
2.0 & -68.98 & 30.30 & -53.13 & 3.61 & -4.34 & -96.15 & -92.55 & -0.52 \\
2.1 & -59.95 & 20.01 & -45.04 & 0.50 & -3.35 & -88.34 & -87.84 & -1.04 \\
2.2 & -52.94 & 13.23 & -38.29 & -1.62 & -2.59 & -80.59 & -82.20 & -1.37 \\
2.3 & -47.35 & 8.76 & -32.61 & -2.98 & -2.00 & -73.21 & -76.19 & -1.53 \\
2.4 & -42.81 & 5.80 & -27.83 & -3.78 & -1.55 & -66.39 & -70.17 & -1.53 \\
2.5 & -39.04 & 3.85 & -23.80 & -4.16 & -1.21 & -60.20 & -64.35 & -1.44 \\
2.6 & -35.85 & 2.55 & -20.40 & -4.23 & -0.94 & -54.64 & -58.87 & -1.29 \\
2.7 & -33.11 & 1.70 & -17.54 & -4.10 & -0.74 & -49.69 & -53.79 & -1.13 \\
2.8 & -30.72 & 1.13 & -15.13 & -3.84 & -0.58 & -45.30 & -49.14 & -0.96 \\
2.9 & -28.61 & 0.75 & -13.09 & -3.49 & -0.46 & -41.42 & -44.91 & -0.80 \\
3.0 & -26.73 & 0.50 & -11.37 & -3.11 & -0.37 & -37.98 & -41.09 & -0.66 \\
3.4 & -20.89 & 0.10 & -6.73 & -1.68 & -0.16 & -27.68 & -29.37 & -0.27 \\
3.8 & -16.80 & 0.02 & -4.22 & -0.78 & -0.08 & -21.08 & -21.86 & -0.10 \\
4.2 & -13.81 & 0.00 & -2.79 & -0.33 & -0.04 & -16.64 & -16.97 & -0.04 \\
4.6 & -11.55 & 0.00 & -1.93 & -0.14 & -0.02 & -13.50 & -13.64 & -0.01 \\
5.0 & -9.81 & 0.00 & -1.38 & -0.06 & -0.01 & -11.20 & -11.26 & -0.00 \\
\hline \hline & & & & & & & & \\
\hline \hline
\end{tabular}




\begin{tabular}{rrrrrrrrrr}
\hline \hline & & \multicolumn{7}{c}{$\mathrm{SAPT} 2+3$} \\
$\mathrm{~d}(\AA)$ & $E_{\text {elst }}^{(1)}$ & $E_{\text {exch }}^{(1)}$ & $E_{\mathrm{IND}}^{[3]}$ & $\delta_{\text {int }}^{\mathrm{HF}}[3]$ & $E_{\mathrm{DISP}}^{[3]}$ & $E_{\text {int }}^{[3]}$ & $E_{\text {int }}^{[3]}+\delta_{\text {int }}^{\mathrm{HF}}[3]$ & $E_{\mathrm{CD}}^{[3]}$ \\
\hline 1.5 & -189.84 & 245.14 & -591.33 & 505.20 & -9.14 & -545.17 & -39.98 & -293.95 \\
1.6 & -146.98 & 161.03 & -350.30 & 274.72 & -11.59 & -347.84 & -73.12 & -138.76 \\
1.7 & -116.97 & 105.90 & -227.48 & 159.98 & -11.31 & -249.86 & -89.87 & -68.33 \\
1.8 & -95.59 & 69.71 & -160.26 & 99.48 & -9.94 & -196.09 & -96.61 & -37.12 \\
1.9 & -80.06 & 45.94 & -119.87 & 64.98 & -8.29 & -162.29 & -97.31 & -23.20 \\
2.0 & -68.53 & 30.30 & -93.17 & 43.64 & -6.71 & -138.10 & -94.46 & -16.57 \\
2.1 & -59.76 & 20.01 & -74.08 & 29.54 & -5.33 & -119.16 & -89.62 & -12.89 \\
2.2 & -52.92 & 13.23 & -59.71 & 19.80 & -4.19 & -103.59 & -83.78 & -10.41 \\
2.3 & -47.46 & 8.76 & -48.55 & 12.95 & -3.27 & -90.52 & -77.57 & -8.46 \\
2.4 & -43.01 & 5.80 & -39.73 & 8.12 & -2.54 & -79.47 & -71.35 & -6.82 \\
2.5 & -39.29 & 3.85 & -32.69 & 4.74 & -1.98 & -70.11 & -65.37 & -5.43 \\
2.6 & -36.13 & 2.55 & -27.06 & 2.42 & -1.54 & -62.17 & -59.75 & -4.27 \\
2.7 & -33.40 & 1.70 & -22.53 & 0.89 & -1.20 & -55.43 & -54.54 & -3.32 \\
2.8 & -31.02 & 1.13 & -18.87 & -0.09 & -0.93 & -49.70 & -49.79 & -2.56 \\
2.9 & -28.91 & 0.75 & -15.91 & -0.68 & -0.73 & -44.80 & -45.48 & -1.95 \\
3.0 & -27.03 & 0.50 & -13.50 & -0.99 & -0.57 & -40.60 & -41.60 & -1.48 \\
3.4 & -21.15 & 0.10 & -7.45 & -0.97 & -0.23 & -28.73 & -29.70 & -0.48 \\
3.8 & -17.02 & 0.02 & -4.49 & -0.51 & -0.10 & -21.59 & -22.10 & -0.15 \\
4.2 & -13.99 & 0.00 & -2.91 & -0.22 & -0.05 & -16.94 & -17.16 & -0.04 \\
4.6 & -11.71 & 0.00 & -1.98 & -0.08 & -0.03 & -13.72 & -13.80 & -0.01 \\
5.0 & -9.94 & 0.00 & -1.41 & -0.03 & -0.02 & -11.36 & -11.39 & -0.00 \\
\hline \hline & & & & & & & &
\end{tabular}




\begin{tabular}{crrrrrrrrr}
\hline \hline \multicolumn{7}{c}{$\mathrm{SAPT}(\mathrm{DFT})\left[\mathrm{PBE} 0 / \mathrm{AC} / S^{2}\right]$} \\
\hline $\mathrm{d}(\AA)$ & $E_{\text {elst }}^{(1)}$ & $E_{\text {exch }}^{(1)}$ & $E_{\text {IND }}^{(2)}$ & $\delta_{\text {int }}^{\mathrm{HF}}[2]$ & $E_{\mathrm{DISP}}^{(2)}$ & $E_{\text {int }}^{[2]}$ & $E_{\text {int }}^{[2]}+\delta_{\text {int }}^{\mathrm{HF}}[2]$ & $E_{\mathrm{CD}}^{(2)}$ & $E_{\mathrm{POL}}^{(2)}$ \\
\hline 1.5 & -190.14 & 232.11 & -160.54 & 83.40 & -14.27 & -132.84 & -49.44 & -122.20 & -38.35 \\
1.6 & -147.21 & 153.28 & -119.09 & 47.31 & -11.67 & -124.70 & -77.39 & -71.79 & -47.30 \\
1.7 & -117.07 & 101.07 & -92.16 & 27.14 & -9.38 & -117.54 & -90.40 & -43.63 & -48.53 \\
1.8 & -95.56 & 66.64 & -75.57 & 16.31 & -7.46 & -111.96 & -95.64 & -29.25 & -46.32 \\
1.9 & -79.90 & 43.95 & -63.51 & 9.54 & -5.89 & -105.36 & -95.82 & -20.80 & -42.71 \\
2.0 & -68.27 & 29.00 & -52.52 & 4.21 & -4.63 & -96.41 & -92.20 & -13.90 & -38.61 \\
2.1 & -59.42 & 19.16 & -44.16 & 0.75 & -3.63 & -88.05 & -87.30 & -9.65 & -34.51 \\
2.2 & -52.53 & 12.66 & -37.78 & -1.18 & -2.84 & -80.47 & -81.65 & -7.18 & -30.60 \\
2.3 & -47.04 & 8.38 & -32.33 & -2.50 & -2.22 & -73.20 & -75.69 & -5.32 & -27.00 \\
2.4 & -42.56 & 5.55 & -27.40 & -3.51 & -1.74 & -66.15 & -69.66 & -3.66 & -23.75 \\
2.5 & -38.84 & 3.68 & -23.26 & -4.09 & -1.36 & -59.78 & -63.88 & -2.43 & -20.84 \\
2.6 & -35.68 & 2.44 & -19.95 & -4.20 & -1.07 & -54.26 & -58.46 & -1.68 & -18.27 \\
2.7 & -32.97 & 1.62 & -17.26 & -4.01 & -0.84 & -49.45 & -53.46 & -1.26 & -16.01 \\
2.8 & -30.59 & 1.08 & -15.02 & -3.72 & -0.67 & -45.20 & -48.92 & -0.95 & -14.07 \\
2.9 & -28.50 & 0.72 & -12.98 & -3.41 & -0.53 & -41.29 & -44.70 & -0.66 & -12.32 \\
3.0 & -26.64 & 0.48 & -11.25 & -3.08 & -0.42 & -37.83 & -40.91 & -0.42 & -10.83 \\
3.4 & -20.82 & 0.09 & -6.70 & -1.67 & -0.18 & -27.61 & -29.27 & -0.10 & -6.60 \\
3.8 & -16.74 & 0.02 & -4.21 & -0.77 & -0.08 & -21.01 & -21.79 & -0.02 & -4.19 \\
4.2 & -13.75 & 0.00 & -2.78 & -0.33 & -0.04 & -16.58 & -16.91 & -0.01 & -2.78 \\
4.6 & -11.51 & 0.00 & -1.92 & -0.14 & -0.02 & -13.45 & -13.59 & -0.00 & -1.92 \\
5.0 & -9.77 & 0.00 & -1.37 & -0.06 & -0.01 & -11.15 & -11.22 & -0.00 & -1.37 \\
\hline \hline & & & & & & & & & \\
\hline
\end{tabular}




\begin{tabular}{rrrrrrrrrrrr}
\hline \hline \multicolumn{10}{c}{$\mathrm{SAPT}(\mathrm{DFT})\left[\mathrm{PBE} / \mathrm{AC} / \mathrm{no} S^{2}\right]$} \\
\hline $\mathrm{d}(\AA)$ & $E_{\text {elst }}^{(1)}$ & $E_{\text {exch }}^{(1)}$ & $E_{\text {IND }}^{(2)}$ & $\delta_{\text {int }}^{\mathrm{HF}}[2]$ & $E_{\mathrm{DISP}}^{(2)}$ & $E_{\text {int }}^{[2]}$ & $E_{\text {int }}^{[2]}+\delta_{\text {int }}^{\mathrm{HF}}[2]$ & $E_{\mathrm{CD}}^{(2)}$ & $E_{\mathrm{POL}}^{(2)}$ & $E_{\mathrm{CD}}^{(2-\infty)}$ & $E_{\mathrm{POL}}^{(2-\infty)}$ \\
\hline 1.5 & -190.14 & 241.69 & -105.21 & 22.90 & -14.27 & -67.92 & -45.02 & -44.18 & -61.03 & -13.26 & -69.05 \\
1.6 & -147.21 & 157.71 & -92.20 & 19.09 & -11.67 & -93.37 & -74.28 & -35.30 & -56.90 & -9.75 & -63.36 \\
1.7 & -117.07 & 103.13 & -80.15 & 14.54 & -9.38 & -103.48 & -88.93 & -27.59 & -52.56 & -8.32 & -57.29 \\
1.8 & -95.56 & 67.59 & -69.21 & 10.03 & -7.46 & -104.65 & -94.62 & -21.20 & -48.02 & -7.97 & -51.22 \\
1.9 & -79.90 & 44.39 & -59.44 & 5.99 & -5.89 & -100.84 & -94.86 & -16.02 & -43.41 & -8.09 & -45.36 \\
2.0 & -68.27 & 29.21 & -50.86 & 2.64 & -4.63 & -94.55 & -91.91 & -11.95 & -38.90 & -8.30 & -39.91 \\
2.1 & -59.42 & 19.25 & -43.43 & 0.05 & -3.63 & -87.22 & -87.17 & -8.80 & -34.63 & -8.40 & -34.97 \\
2.2 & -52.53 & 12.71 & -37.05 & -1.82 & -2.84 & -79.70 & -81.53 & -6.40 & -30.65 & -8.32 & -30.55 \\
2.3 & -47.04 & 8.40 & -31.63 & -3.08 & -2.22 & -72.48 & -75.56 & -4.61 & -27.02 & -8.07 & -26.64 \\
2.4 & -42.56 & 5.56 & -27.04 & -3.82 & -1.74 & -65.77 & -69.60 & -3.28 & -23.76 & -7.64 & -23.22 \\
2.5 & -38.84 & 3.68 & -23.16 & -4.18 & -1.36 & -59.68 & -63.86 & -2.32 & -20.84 & -7.10 & -20.24 \\
2.6 & -35.68 & 2.44 & -19.90 & -4.24 & -1.07 & -54.21 & -58.45 & -1.63 & -18.27 & -6.48 & -17.67 \\
2.7 & -32.97 & 1.62 & -17.15 & -4.11 & -0.84 & -49.34 & -53.45 & -1.14 & -16.01 & -5.82 & -15.44 \\
2.8 & -30.59 & 1.08 & -14.87 & -3.84 & -0.67 & -45.05 & -48.90 & -0.80 & -14.07 & -5.19 & -13.52 \\
2.9 & -28.50 & 0.72 & -12.87 & -3.50 & -0.53 & -41.19 & -44.68 & -0.55 & -12.32 & -4.50 & -11.87 \\
3.0 & -26.64 & 0.48 & -11.21 & -3.11 & -0.42 & -37.79 & -40.91 & -0.38 & -10.83 & -3.88 & -10.44 \\
3.4 & -20.82 & 0.09 & -6.68 & -1.68 & -0.18 & -27.59 & -29.27 & -0.08 & -6.60 & -1.94 & -6.43 \\
3.8 & -16.74 & 0.02 & -4.21 & -0.78 & -0.08 & -21.01 & -21.79 & -0.02 & -4.19 & -0.84 & -4.14 \\
4.2 & -13.75 & 0.00 & -2.78 & -0.33 & -0.04 & -16.58 & -16.91 & -0.00 & -2.78 & -0.34 & -2.78 \\
4.6 & -11.51 & 0.00 & -1.92 & -0.14 & -0.02 & -13.45 & -13.59 & -0.00 & -1.92 & -0.13 & -1.93 \\
5.0 & -9.77 & 0.00 & -1.37 & -0.06 & -0.01 & -11.15 & -11.22 & -0.00 & -1.37 & -0.05 & -1.38 \\
\hline \hline
\end{tabular}




\section{$7.3 \quad \mathrm{~F}^{-} \ldots \mathrm{H}_{2} \mathrm{O}$}

\begin{tabular}{crrrr}
\hline \hline \multicolumn{5}{c}{$E_{\text {int }}$} \\
\hline $\mathrm{d}(\AA)$ & $\mathrm{CCSD}(\mathrm{T})$ & B3LYP & PBE0 & $\omega$ B97X-D \\
\hline 0.7 & 161.48 & 161.21 & 157.01 & 158.97 \\
0.8 & 70.72 & 70.78 & 66.66 & 68.78 \\
0.9 & 21.67 & 21.77 & 17.94 & 20.15 \\
1.0 & -5.16 & -5.13 & -8.54 & -6.36 \\
1.1 & -19.70 & -19.77 & -22.71 & -20.69 \\
1.2 & -27.20 & -27.38 & -29.85 & -28.07 \\
1.3 & -30.62 & -30.88 & -32.92 & -31.41 \\
1.4 & -31.61 & -31.94 & -33.60 & -32.37 \\
1.5 & -31.18 & -31.55 & -32.89 & -31.92 \\
1.6 & -29.94 & -30.33 & -31.42 & -30.64 \\
2.1 & -20.91 & -21.20 & -21.65 & -21.29 \\
2.6 & -13.92 & -14.11 & -14.37 & -14.09 \\
3.1 & -9.59 & -9.73 & -9.91 & -9.70 \\
3.6 & -6.93 & -7.02 & -7.15 & -7.04 \\
4.1 & -5.23 & -5.29 & -5.38 & -5.33 \\
4.6 & -4.10 & -4.14 & -4.19 & -4.17 \\
5.1 & -3.30 & -3.33 & -3.36 & -3.35 \\
\hline \hline
\end{tabular}




\begin{tabular}{|c|c|c|c|c|c|c|c|c|}
\hline \multirow[b]{2}{*}{$\mathrm{d}(\AA)$} & \multicolumn{4}{|c|}{ PBE0||ALMO } & \multicolumn{4}{|c|}{$\omega B 97 X-D \| A L M O$} \\
\hline & $E_{\text {elst }}+E_{\text {exch }}$ & $E_{\text {pol }}$ & $E_{\mathrm{cd}}$ & $E_{\text {int }}$ & $E_{\text {elst }}+E_{\text {exch }}$ & $E_{\mathrm{pol}}$ & $E_{\mathrm{cd}}$ & $E_{\text {int }}$ \\
\hline 0.7 & 393.74 & -193.43 & -43.29 & 157.01 & 396.93 & -194.70 & -43.26 & 158.97 \\
\hline 0.8 & 244.87 & -138.55 & -39.66 & 66.66 & 247.57 & -139.25 & -39.54 & 68.78 \\
\hline 0.9 & 151.31 & -98.56 & -34.82 & 17.94 & 153.46 & -98.75 & -34.55 & 20.15 \\
\hline 1.0 & 91.30 & -70.28 & -29.56 & -8.54 & 92.94 & -70.11 & -29.19 & -6.36 \\
\hline 1.1 & 52.29 & -50.58 & -24.42 & -22.71 & 53.52 & -50.25 & -23.97 & -20.69 \\
\hline 1.2 & 26.80 & -36.86 & -19.80 & -29.85 & 27.71 & -36.50 & -19.28 & -28.07 \\
\hline 1.3 & 10.23 & -27.21 & -15.94 & -32.92 & 10.89 & -26.91 & -15.39 & -31.41 \\
\hline 1.4 & -0.41 & -20.36 & -12.83 & -33.60 & 0.09 & -20.14 & -12.31 & -32.37 \\
\hline 1.5 & -7.04 & -15.49 & -10.37 & -32.89 & -6.67 & -15.34 & -9.90 & -31.92 \\
\hline 1.6 & -11.01 & -12.01 & -8.40 & -31.42 & -10.72 & -11.91 & -8.02 & -30.64 \\
\hline 2.1 & -14.02 & -4.55 & -3.08 & -21.65 & -13.88 & -4.49 & -2.92 & -21.29 \\
\hline 2.6 & -10.82 & -2.38 & -1.17 & -14.37 & -10.65 & -2.34 & -1.09 & -14.09 \\
\hline 3.1 & -8.02 & -1.38 & -0.51 & -9.91 & -7.97 & -1.38 & -0.35 & -9.70 \\
\hline 3.6 & -6.11 & -0.84 & -0.20 & -7.15 & -6.07 & -0.85 & -0.12 & -7.04 \\
\hline 4.1 & -4.72 & -0.54 & -0.12 & -5.38 & -4.83 & -0.54 & 0.04 & -5.33 \\
\hline 4.6 & -3.82 & -0.36 & -0.01 & -4.19 & -4.16 & -0.37 & 0.36 & -4.17 \\
\hline 5.1 & -3.09 & -0.25 & -0.03 & -3.36 & -3.49 & -0.26 & 0.39 & -3.35 \\
\hline
\end{tabular}

\begin{tabular}{crrrrrrrr}
\hline \hline & \multicolumn{3}{c}{ B3LYP\|CSOV } & \multicolumn{5}{c}{ HF||RVS } \\
\cline { 2 - 3 } \cline { 6 - 8 } $\mathrm{d}(\AA)$ & $E_{\text {pol }}$ & $E_{\text {cd }}$ & & $E_{\text {elst }}$ & $E_{\text {exch }}$ & $E_{\text {pol }}$ & $E_{\text {cd }}$ & $E_{\text {int }}$ \\
\hline 0.7 & -180.25 & -52.05 & & -66.46 & 459.97 & -197.59 & -47.02 & 156.25 \\
0.8 & -129.58 & -45.64 & & -95.75 & 340.17 & -140.15 & -40.34 & 66.86 \\
0.9 & -91.88 & -39.41 & & -97.26 & 248.15 & -98.07 & -34.04 & 19.20 \\
1.0 & -64.84 & -33.53 & & -88.27 & 179.23 & -68.45 & -28.18 & -6.40 \\
1.1 & -46.20 & -27.84 & & -76.48 & 128.57 & -48.37 & -22.60 & -20.00 \\
1.2 & -33.59 & -22.55 & & -65.07 & 91.81 & -34.95 & -17.51 & -26.88 \\
1.3 & -24.96 & -18.02 & & -55.11 & 65.38 & -25.89 & -13.25 & -29.89 \\
1.4 & -18.91 & -14.37 & & -46.80 & 46.52 & -19.62 & -9.92 & -30.68 \\
1.5 & -14.59 & -11.53 & & -39.98 & 33.11 & -15.16 & -7.42 & -30.17 \\
1.6 & -11.43 & -9.34 & & -34.43 & 23.60 & -11.93 & -5.60 & -28.94 \\
2.1 & -4.44 & -3.56 & & -18.58 & 4.53 & -4.58 & -1.54 & -20.38 \\
2.6 & -2.39 & -1.42 & & -11.93 & 0.94 & -2.31 & -0.43 & -13.81 \\
3.1 & -1.42 & -0.63 & & -8.45 & 0.20 & -1.31 & -0.11 & -9.70 \\
3.6 & -0.88 & -0.32 & & -6.34 & 0.04 & -0.80 & -0.03 & -7.13 \\
4.1 & -0.57 & -0.19 & & -4.94 & 0.01 & -0.51 & -0.00 & -5.44 \\
4.6 & -0.38 & -0.13 & & -3.96 & 0.00 & -0.34 & -0.00 & -4.30 \\
5.1 & -0.26 & -0.09 & -3.25 & 0.00 & -0.23 & -0.00 & -3.48 \\
\hline \hline
\end{tabular}




\begin{tabular}{rrrrrrrrr}
\hline \hline \multicolumn{7}{c}{ SAPT2 } \\
\hline $\mathrm{d}(\AA)$ & $E_{\text {elst }}^{(1)}$ & $E_{\text {exch }}^{(1)}$ & $E_{\text {IND }}^{(2)}$ & $\delta_{\text {int }}^{\mathrm{HF}}[2]$ & $E_{\text {DISP }}^{(2)}$ & $E_{\text {int }}^{[2]}$ & $E_{\text {int }}^{[2]}+\delta_{\text {int }}^{\mathrm{HF}}[2]$ & $E_{\mathrm{CD}}^{(2)}$ \\
\hline 0.7 & -60.85 & 423.47 & -258.87 & 44.17 & -40.76 & 62.98 & 107.15 & -58.28 \\
0.8 & -91.44 & 323.70 & -159.30 & -5.29 & -30.81 & 42.15 & 36.86 & -45.56 \\
0.9 & -94.42 & 243.76 & -101.25 & -23.32 & -23.46 & 24.63 & 1.31 & -34.72 \\
1.0 & -86.68 & 181.60 & -67.13 & -26.69 & -18.05 & 9.74 & -16.95 & -25.63 \\
1.1 & -75.73 & 134.29 & -46.66 & -24.11 & -14.02 & -2.12 & -26.23 & -18.52 \\
1.2 & -64.77 & 98.82 & -33.98 & -19.69 & -10.99 & -10.93 & -30.62 & -13.32 \\
1.3 & -55.00 & 72.49 & -25.80 & -15.26 & -8.69 & -17.00 & -32.26 & -9.70 \\
1.4 & -46.73 & 53.08 & -20.27 & -11.46 & -6.92 & -20.84 & -32.29 & -7.23 \\
1.5 & -39.90 & 38.85 & -16.35 & -8.44 & -5.54 & -22.94 & -31.37 & -5.52 \\
1.6 & -34.30 & 28.43 & -13.44 & -6.14 & -4.46 & -23.78 & -29.91 & -4.30 \\
2.1 & -18.31 & 6.09 & -5.92 & -1.15 & -1.61 & -19.75 & -20.90 & -1.30 \\
2.6 & -11.63 & 1.39 & -2.96 & -0.19 & -0.63 & -13.83 & -14.02 & -0.39 \\
3.1 & -8.17 & 0.34 & -1.60 & -0.02 & -0.26 & -9.69 & -9.72 & -0.13 \\
3.6 & -6.10 & 0.09 & -0.93 & 0.00 & -0.11 & -7.05 & -7.05 & -0.04 \\
4.1 & -4.73 & 0.02 & -0.58 & 0.01 & -0.05 & -5.34 & -5.34 & -0.01 \\
4.6 & -3.79 & 0.01 & -0.38 & 0.00 & -0.03 & -4.19 & -4.18 & -0.01 \\
5.1 & -3.10 & 0.00 & -0.26 & 0.00 & -0.01 & -3.37 & -3.37 & -0.00 \\
\hline \hline
\end{tabular}

SAPT $2+3$

\begin{tabular}{rrrrrrrrrr}
\hline $\mathrm{d}(\AA)$ & $E_{\text {elst }}^{(1)}$ & $E_{\text {exch }}^{(1)}$ & $E_{\text {IND }}^{[3]}$ & $\delta_{\text {int }}^{\mathrm{HF}}[3]$ & $E_{\text {DISP }}^{[3]}$ & $E_{\text {int }}^{[3]}$ & $E_{\text {int }}^{[3]}+\delta_{\text {int }}^{\mathrm{HF}}[3]$ & $E_{\mathrm{CD}}^{[3]}$ \\
\hline 0.7 & -67.14 & 423.47 & -602.45 & 387.74 & -52.55 & -298.66 & 89.08 & -213.69 \\
0.8 & -95.74 & 323.70 & -342.60 & 178.00 & -38.41 & -153.04 & 24.96 & -152.57 \\
0.9 & -97.20 & 243.76 & -199.29 & 74.72 & -28.60 & -81.32 & -6.60 & -102.67 \\
1.0 & -88.34 & 181.60 & -120.36 & 26.53 & -21.79 & -48.89 & -22.35 & -66.27 \\
1.1 & -76.62 & 134.29 & -76.25 & 5.48 & -16.95 & -35.53 & -30.04 & -42.04 \\
1.2 & -65.13 & 98.82 & -50.91 & -2.76 & -13.41 & -30.64 & -33.40 & -26.83 \\
1.3 & -55.02 & 72.49 & -35.80 & -5.26 & -10.75 & -29.08 & -34.34 & -17.52 \\
1.4 & -46.54 & 53.08 & -26.37 & -5.36 & -8.71 & -28.53 & -33.88 & -11.82 \\
1.5 & -39.59 & 38.85 & -20.17 & -4.61 & -7.10 & -28.01 & -32.62 & -8.23 \\
1.6 & -33.94 & 28.43 & -14.97 & -3.68 & -5.82 & -26.29 & -30.90 & -5.89 \\
2.1 & -18.04 & 6.09 & -6.20 & -0.87 & -2.25 & -20.40 & -21.27 & -1.54 \\
2.6 & -11.49 & 1.39 & -2.96 & -0.19 & -0.91 & -13.98 & -14.17 & -0.43 \\
3.1 & -8.10 & 0.34 & -1.58 & -0.05 & -0.38 & -9.72 & -9.77 & -0.14 \\
3.6 & -6.06 & 0.09 & -0.91 & -0.01 & -0.16 & -7.05 & -7.06 & -0.05 \\
4.1 & -4.71 & 0.02 & -0.57 & -0.00 & -0.08 & -5.33 & -5.34 & -0.01 \\
4.6 & -3.78 & 0.01 & -0.37 & -0.00 & -0.04 & -4.18 & -4.18 & -0.01 \\
5.1 & -3.09 & 0.00 & -0.26 & -0.00 & -0.02 & -3.37 & -3.37 & -0.00 \\
\hline \hline
\end{tabular}




\begin{tabular}{crrrrrrrrr}
\hline \hline \multicolumn{10}{c}{ SAPT(DFT)[PBE0/AC/ $\left.S^{2}\right]$} \\
\hline $\mathrm{d}(\AA)$ & $E_{\text {elst }}^{(1)}$ & $E_{\text {exch }}^{(1)}$ & $E_{\text {IND }}^{(2)}$ & $\delta_{\text {int }}^{\mathrm{HF}}[2]$ & $E_{\text {DISP }}^{(2)}$ & $E_{\text {int }}^{[2]}$ & $E_{\text {int }}^{[2]}+\delta_{\text {int }}^{\mathrm{HF}}[2]$ & $E_{\text {CD }}^{(2)}$ & $E_{\text {POL }}^{(2)}$ \\
\hline 0.7 & -70.30 & 343.23 & -244.88 & 121.29 & -35.09 & -7.05 & 114.24 & -256.16 & 11.28 \\
0.8 & -96.93 & 272.34 & -149.98 & 42.12 & -27.46 & -2.02 & 40.10 & -144.55 & -5.43 \\
0.9 & -97.47 & 211.14 & -94.20 & 4.90 & -21.66 & -2.18 & 2.73 & -81.09 & -13.11 \\
1.0 & -88.20 & 160.77 & -61.29 & -10.28 & -17.25 & -5.98 & -16.25 & -45.43 & -15.86 \\
1.1 & -76.33 & 120.80 & -41.67 & -14.69 & -13.87 & -11.07 & -25.76 & -25.60 & -16.07 \\
1.2 & -64.81 & 89.90 & -29.66 & -14.35 & -11.23 & -15.80 & -30.16 & -14.58 & -15.07 \\
1.3 & -54.72 & 66.46 & -22.08 & -12.24 & -9.16 & -19.49 & -31.73 & -8.46 & -13.62 \\
1.4 & -46.28 & 48.93 & -17.14 & -9.73 & -7.50 & -21.99 & -31.72 & -5.06 & -12.08 \\
1.5 & -39.35 & 35.94 & -13.76 & -7.44 & -6.17 & -23.35 & -30.78 & -3.13 & -10.63 \\
1.6 & -33.73 & 26.36 & -11.33 & -5.54 & -5.10 & -23.79 & -29.33 & -2.02 & -9.31 \\
2.1 & -17.87 & 5.66 & -5.18 & -1.08 & -2.04 & -19.43 & -20.52 & -0.36 & -4.82 \\
2.6 & -11.34 & 1.29 & -2.76 & -0.14 & -0.85 & -13.66 & -13.80 & -0.14 & -2.62 \\
3.1 & -7.99 & 0.32 & -1.60 & 0.05 & -0.36 & -9.64 & -9.58 & -0.11 & -1.49 \\
3.6 & -5.97 & 0.08 & -0.93 & 0.03 & -0.16 & -6.98 & -6.95 & -0.06 & -0.88 \\
4.1 & -4.64 & 0.02 & -0.60 & 0.03 & -0.07 & -5.29 & -5.26 & -0.05 & -0.55 \\
4.6 & -3.72 & 0.01 & -0.40 & 0.02 & -0.04 & -4.15 & -4.13 & -0.04 & -0.36 \\
5.1 & -3.05 & 0.00 & -0.28 & 0.02 & -0.02 & -3.35 & -3.33 & -0.04 & -0.25 \\
\hline \hline
\end{tabular}

\begin{tabular}{rrrrrrrrrrrr}
\hline \hline \multicolumn{10}{c}{$\mathrm{SAPT}(\mathrm{DFT})\left[\mathrm{PBE} 0 / \mathrm{AC} / \mathrm{no} S^{2}\right]$} \\
\hline $\mathrm{d}(\AA)$ & $E_{\text {elst }}^{(1)}$ & $E_{\text {exch }}^{(1)}$ & $E_{\mathrm{IND}}^{(2)}$ & $\delta_{\text {int }}^{\mathrm{HF}}[2]$ & $E_{\mathrm{DISP}}^{(2)}$ & $E_{\text {int }}^{[2]}$ & $E_{\text {int }}^{[2]}+\delta_{\text {int }}^{\mathrm{HF}}[2]$ & $E_{\mathrm{CD}}^{(2)}$ & $E_{\mathrm{POL}}^{(2)}$ & $E_{\mathrm{CD}}^{(2-\infty)}$ & $E_{\mathrm{POL}}^{(2-\infty)}$ \\
\hline 0.7 & -70.30 & 427.19 & -101.71 & -87.23 & -35.09 & 220.09 & 132.85 & -74.03 & -27.68 & -117.21 & -71.73 \\
0.8 & -96.93 & 323.88 & -72.11 & -73.93 & -27.46 & 127.39 & 53.46 & -46.08 & -26.03 & -87.51 & -58.53 \\
0.9 & -97.47 & 241.81 & -52.40 & -58.46 & -21.66 & 70.29 & 11.82 & -28.47 & -23.93 & -63.26 & -47.61 \\
1.0 & -88.20 & 178.60 & -39.04 & -44.42 & -17.25 & 34.10 & -10.31 & -17.58 & -21.47 & -44.74 & -38.72 \\
1.1 & -76.33 & 131.00 & -29.85 & -32.93 & -13.87 & 10.96 & -21.98 & -10.93 & -18.92 & -31.23 & -31.55 \\
1.2 & -64.81 & 95.67 & -23.38 & -24.05 & -11.23 & -3.76 & -27.81 & -6.89 & -16.49 & -21.64 & -25.79 \\
1.3 & -54.72 & 69.70 & -18.74 & -17.38 & -9.16 & -12.91 & -30.30 & -4.43 & -14.31 & -14.96 & -21.17 \\
1.4 & -46.28 & 50.74 & -15.32 & -12.49 & -7.50 & -18.35 & -30.84 & -2.91 & -12.41 & -10.37 & -17.44 \\
1.5 & -39.35 & 36.95 & -12.73 & -8.93 & -6.17 & -21.31 & -30.24 & -1.96 & -10.77 & -7.22 & -14.44 \\
1.6 & -33.73 & 26.93 & -10.72 & -6.37 & -5.10 & -22.62 & -28.99 & -1.34 & -9.37 & -5.08 & -12.00 \\
2.1 & -17.87 & 5.69 & -5.10 & -1.15 & -2.04 & -19.32 & -20.47 & -0.28 & -4.82 & -1.11 & -5.13 \\
2.6 & -11.34 & 1.29 & -2.68 & -0.19 & -0.85 & -13.59 & -13.78 & -0.07 & -2.62 & -0.41 & -2.47 \\
3.1 & -7.99 & 0.32 & -1.50 & -0.02 & -0.36 & -9.54 & -9.56 & -0.02 & -1.49 & -0.20 & -1.32 \\
3.6 & -5.97 & 0.08 & -0.89 & 0.00 & -0.16 & -6.94 & -6.93 & -0.02 & -0.88 & -0.12 & -0.77 \\
4.1 & -4.64 & 0.02 & -0.56 & 0.01 & -0.07 & -5.26 & -5.25 & -0.02 & -0.55 & -0.07 & -0.48 \\
4.6 & -3.72 & 0.01 & -0.37 & 0.00 & -0.04 & -4.12 & -4.12 & -0.01 & -0.36 & -0.05 & -0.32 \\
5.1 & -3.05 & 0.00 & -0.25 & 0.00 & -0.02 & -3.32 & -3.32 & -0.01 & -0.25 & -0.03 & -0.22 \\
\hline \hline
\end{tabular}




\section{$7.4 \mathrm{Cl}^{-} \ldots \mathrm{H}_{2} \mathrm{O}$}

\begin{tabular}{rrrrr}
\hline \hline \multicolumn{5}{c}{$E_{\text {int }}$} \\
\hline $\mathrm{d}(\AA)$ & $\mathrm{CCSD}(\mathrm{T})$ & B3LYP & PBE0 & $\omega \mathrm{B} 97 \mathrm{X}-\mathrm{D}$ \\
\hline 1.3 & 38.53 & 37.62 & 34.24 & 37.02 \\
1.4 & 19.41 & 18.88 & 15.84 & 18.23 \\
1.5 & 6.50 & 6.23 & 3.55 & 5.64 \\
1.6 & -2.13 & -2.21 & -4.54 & -2.73 \\
1.7 & -7.78 & -7.73 & -9.73 & -8.19 \\
1.8 & -11.36 & -11.22 & -12.93 & -11.64 \\
1.9 & -13.49 & -13.30 & -14.74 & -13.68 \\
2.0 & -14.62 & -14.38 & -15.61 & -14.75 \\
2.1 & -15.06 & -14.80 & -15.84 & -15.14 \\
2.2 & -15.03 & -14.77 & -15.65 & -15.09 \\
2.3 & -14.71 & -14.44 & -15.20 & -14.74 \\
2.8 & -11.48 & -11.29 & -11.68 & -11.47 \\
3.3 & -8.45 & -8.32 & -8.57 & -8.45 \\
3.8 & -6.32 & -6.23 & -6.40 & -6.35 \\
4.3 & -4.87 & -4.82 & -4.93 & -4.91 \\
4.8 & -3.87 & -3.84 & -3.91 & -3.91 \\
5.3 & -3.16 & -3.14 & -3.18 & -3.20 \\
\hline \hline
\end{tabular}




\begin{tabular}{|c|c|c|c|c|c|c|c|c|c|}
\hline \multirow[b]{2}{*}{$\mathrm{d}(\AA)$} & \multicolumn{4}{|c|}{ PBE0||ALMO } & \multicolumn{5}{|c|}{$\omega B 97 X-D \|$ ALMO } \\
\hline & $E_{\text {elst }}+E_{\text {exch }}$ & $E_{\mathrm{pol}}$ & $E_{\mathrm{cd}}$ & $E_{\text {int }}$ & $E_{\text {elst }}$ & $+E_{\text {exch }}$ & $E_{\mathrm{pol}}$ & $E_{\text {cd }}$ & $E_{\text {int }}$ \\
\hline 1.3 & 98.52 & -34.48 & -29.80 & 34.24 & & 100.52 & -34.24 & -29.27 & 37.02 \\
\hline 1.4 & 65.01 & -24.66 & -24.52 & 15.84 & & 66.68 & -24.52 & -23.92 & 18.23 \\
\hline 1.5 & 41.38 & -17.95 & -19.88 & 3.55 & & 42.79 & -17.87 & -19.28 & 5.64 \\
\hline 1.6 & 24.87 & -13.41 & -16.00 & -4.54 & & 25.97 & -13.37 & -15.34 & -2.73 \\
\hline 1.7 & 13.30 & -10.33 & -12.70 & -9.73 & & 14.19 & -10.29 & -12.09 & -8.19 \\
\hline 1.8 & 5.30 & -8.19 & -10.04 & -12.93 & & 6.00 & -8.15 & -9.49 & -11.64 \\
\hline 1.9 & -0.14 & -6.66 & -7.95 & -14.74 & & 0.37 & -6.61 & -7.44 & -13.68 \\
\hline 2.0 & -3.85 & -5.51 & -6.25 & -15.61 & & -3.44 & -5.46 & -5.84 & -14.75 \\
\hline 2.1 & -6.25 & -4.63 & -4.96 & -15.84 & & -5.98 & -4.58 & -4.59 & -15.14 \\
\hline 2.2 & -7.74 & -3.93 & -3.98 & -15.65 & & -7.62 & -3.88 & -3.59 & -15.09 \\
\hline 2.3 & -8.65 & -3.38 & -3.17 & -15.20 & & -8.60 & -3.33 & -2.81 & -14.74 \\
\hline 2.8 & -8.90 & -1.77 & -1.01 & -11.68 & & -8.75 & -1.73 & -0.99 & -11.47 \\
\hline 3.3 & -7.04 & -1.06 & -0.47 & -8.57 & & -6.96 & -1.04 & -0.45 & -8.45 \\
\hline 3.8 & -5.48 & -0.66 & -0.26 & -6.40 & & -5.33 & -0.66 & -0.35 & -6.35 \\
\hline 4.3 & -4.32 & -0.43 & -0.18 & -4.93 & & -4.51 & -0.43 & 0.03 & -4.91 \\
\hline 4.8 & -3.62 & -0.29 & -0.00 & -3.91 & & -4.48 & -0.30 & 0.87 & -3.91 \\
\hline 5.3 & -2.97 & -0.20 & -0.01 & -3.18 & & -4.00 & -0.23 & 1.03 & -3.20 \\
\hline
\end{tabular}

SAPT2

\begin{tabular}{rrrrrrrrrr}
\hline $\mathrm{d}(\AA)$ & $E_{\text {elst }}^{(1)}$ & $E_{\text {exch }}^{(1)}$ & $E_{\text {IND }}^{(2)}$ & $\delta_{\text {int }}^{\mathrm{HF}}[2]$ & $E_{\text {DISP }}^{(2)}$ & $E_{\text {int }}^{[2]}$ & $E_{\text {int }}^{[2]}+\delta_{\text {int }}^{\mathrm{HF}}[2]$ & $E_{\mathrm{CD}}^{(2)}$ \\
\hline 1.3 & -64.68 & 163.00 & -54.34 & 0.34 & -20.28 & 23.70 & 24.04 & -20.86 \\
1.4 & -55.66 & 123.63 & -35.62 & -5.89 & -16.41 & 15.95 & 10.05 & -14.90 \\
1.5 & -47.56 & 93.47 & -24.23 & -7.86 & -13.31 & 8.37 & 0.51 & -10.69 \\
1.6 & -40.61 & 70.48 & -17.17 & -7.80 & -10.83 & 1.87 & -5.93 & -7.74 \\
1.7 & -34.78 & 53.06 & -12.71 & -6.90 & -8.86 & -3.29 & -10.19 & -5.66 \\
1.8 & -29.96 & 39.90 & -9.79 & -5.75 & -7.27 & -7.12 & -12.87 & -4.20 \\
1.9 & -26.00 & 29.99 & -7.80 & -4.63 & -6.00 & -9.81 & -14.44 & -3.15 \\
2.0 & -22.74 & 22.55 & -6.40 & -3.65 & -4.97 & -11.56 & -15.21 & -2.39 \\
2.1 & -20.06 & 16.96 & -5.36 & -2.83 & -4.13 & -12.59 & -15.43 & -1.82 \\
2.2 & -17.83 & 12.76 & -4.57 & -2.18 & -3.44 & -13.08 & -15.26 & -1.40 \\
2.3 & -15.98 & 9.62 & -3.94 & -1.67 & -2.88 & -13.18 & -14.85 & -1.08 \\
2.8 & -10.19 & 2.39 & -2.08 & -0.43 & -1.21 & -11.10 & -11.52 & -0.32 \\
3.3 & -7.27 & 0.62 & -1.19 & -0.11 & -0.54 & -8.38 & -8.49 & -0.11 \\
3.8 & -5.54 & 0.17 & -0.71 & -0.03 & -0.25 & -6.33 & -6.36 & -0.04 \\
4.3 & -4.38 & 0.05 & -0.45 & -0.01 & -0.12 & -4.91 & -4.91 & -0.02 \\
4.8 & -3.56 & 0.01 & -0.30 & -0.00 & -0.07 & -3.91 & -3.91 & -0.01 \\
5.3 & -2.95 & 0.00 & -0.21 & -0.00 & -0.04 & -3.19 & -3.19 & -0.00 \\
\hline \hline
\end{tabular}




\begin{tabular}{rrrrrrrrrr}
\hline \hline \multicolumn{7}{c}{ SAPT2+3 } \\
\hline $\mathrm{d}(\AA)$ & $E_{\text {elst }}^{(1)}$ & $E_{\text {exch }}^{(1)}$ & $E_{\text {IND }}^{[3]}$ & $\delta_{\text {int }}^{\mathrm{HF}}[3]$ & $E_{\text {DISP }}^{[3]}$ & $E_{\text {int }}^{[3]}$ & $E_{\text {int }}^{[3]}+\delta_{\text {int }}^{\mathrm{HF}}[3]$ & $E_{\mathrm{CD}}^{[3]}$ \\
\hline 1.3 & -63.47 & 163.00 & -141.00 & 87.01 & -23.13 & -64.60 & 22.40 & -73.49 \\
1.4 & -54.59 & 123.63 & -84.02 & 42.50 & -18.76 & -33.74 & 8.77 & -46.40 \\
1.5 & -46.65 & 93.47 & -51.69 & 19.60 & -15.22 & -20.08 & -0.48 & -29.64 \\
1.6 & -39.85 & 70.48 & -33.10 & 8.13 & -12.39 & -14.86 & -6.73 & -19.23 \\
1.7 & -34.16 & 53.06 & -22.20 & 2.60 & -10.14 & -13.44 & -10.84 & -12.68 \\
1.8 & -29.46 & 39.90 & -15.62 & 0.09 & -8.34 & -13.52 & -13.43 & -8.51 \\
1.9 & -25.59 & 29.99 & -11.51 & -0.92 & -6.89 & -14.00 & -14.93 & -5.80 \\
2.0 & -22.42 & 22.55 & -8.83 & -1.22 & -5.73 & -14.43 & -15.65 & -4.03 \\
2.1 & -19.81 & 16.96 & -7.00 & -1.20 & -4.77 & -14.62 & -15.82 & -2.84 \\
2.2 & -17.64 & 12.76 & -5.70 & -1.05 & -3.99 & -14.57 & -15.62 & -2.03 \\
2.3 & -15.84 & 9.62 & -4.74 & -0.87 & -3.35 & -14.31 & -15.18 & -1.48 \\
2.8 & -10.17 & 2.39 & -2.25 & -0.25 & -1.43 & -11.47 & -11.72 & -0.39 \\
3.3 & -7.29 & 0.62 & -1.23 & -0.07 & -0.63 & -8.54 & -8.61 & -0.13 \\
3.8 & -5.56 & 0.17 & -0.73 & -0.02 & -0.29 & -6.42 & -6.43 & -0.06 \\
4.3 & -4.41 & 0.05 & -0.45 & -0.01 & -0.14 & -4.96 & -4.96 & -0.02 \\
4.8 & -3.59 & 0.01 & -0.30 & -0.00 & -0.07 & -3.94 & -3.95 & -0.01 \\
5.3 & -2.98 & 0.00 & -0.21 & -0.00 & -0.04 & -3.22 & -3.22 & -0.00 \\
\hline \hline
\end{tabular}

\begin{tabular}{crrrrrrrrr}
\hline \hline \multicolumn{10}{c}{$\mathrm{SAPT}(\mathrm{DFT})\left[\mathrm{PBE} / \mathrm{AC} / S^{2}\right]$} \\
\hline $\mathrm{d}(\AA)$ & $E_{\text {elst }}^{(1)}$ & $E_{\text {exch }}^{(1)}$ & $E_{\text {IND }}^{(2)}$ & $\delta_{\text {int }}^{\mathrm{HF}}[2]$ & $E_{\mathrm{DISP}}^{(2)}$ & $E_{\text {int }}^{[2]}$ & $E_{\text {int }}^{[2]}+\delta_{\text {int }}^{\mathrm{HF}}[2]$ & $E_{\mathrm{CD}}^{(2)}$ & $E_{\mathrm{POL}}^{(2)}$ \\
\hline 1.3 & -64.91 & 146.69 & -53.30 & 18.05 & -19.59 & 8.88 & 26.93 & -38.77 & -14.53 \\
1.4 & -55.71 & 113.29 & -34.70 & 4.74 & -16.14 & 6.73 & 11.47 & -23.12 & -11.58 \\
1.5 & -47.49 & 86.87 & -23.40 & -1.49 & -13.32 & 2.65 & 1.16 & -13.96 & -9.45 \\
1.6 & -40.45 & 66.24 & -16.46 & -3.96 & -11.02 & -1.69 & -5.65 & -8.58 & -7.88 \\
1.7 & -34.56 & 50.29 & -12.12 & -4.54 & -9.15 & -5.54 & -10.08 & -5.42 & -6.70 \\
1.8 & -29.71 & 38.06 & -9.29 & -4.27 & -7.62 & -8.55 & -12.82 & -3.51 & -5.78 \\
1.9 & -25.72 & 28.75 & -7.38 & -3.69 & -6.36 & -10.72 & -14.41 & -2.34 & -5.05 \\
2.0 & -22.46 & 21.69 & -6.02 & -3.06 & -5.33 & -12.13 & -15.19 & -1.58 & -4.44 \\
2.1 & -19.78 & 16.36 & -5.02 & -2.47 & -4.48 & -12.93 & -15.40 & -1.09 & -3.93 \\
2.2 & -17.57 & 12.34 & -4.26 & -1.96 & -3.77 & -13.27 & -15.23 & -0.77 & -3.50 \\
2.3 & -15.73 & 9.31 & -3.69 & -1.52 & -3.18 & -13.30 & -14.82 & -0.56 & -3.12 \\
2.8 & -10.03 & 2.31 & -2.06 & -0.33 & -1.39 & -11.17 & -11.50 & -0.22 & -1.85 \\
3.3 & -7.17 & 0.60 & -1.27 & 0.01 & -0.63 & -8.47 & -8.46 & -0.16 & -1.11 \\
3.8 & -5.46 & 0.16 & -0.77 & 0.04 & -0.29 & -6.37 & -6.33 & -0.09 & -0.68 \\
4.3 & -4.33 & 0.05 & -0.50 & 0.04 & -0.14 & -4.92 & -4.88 & -0.07 & -0.43 \\
4.8 & -3.52 & 0.01 & -0.35 & 0.05 & -0.07 & -3.93 & -3.88 & -0.07 & -0.28 \\
5.3 & -2.92 & 0.00 & -0.24 & 0.03 & -0.04 & -3.20 & -3.17 & -0.04 & -0.20 \\
\hline \hline
\end{tabular}




\begin{tabular}{crrrrrrrrrr}
\hline \hline \multicolumn{10}{c}{$\mathrm{SAPT}(\mathrm{DFT})\left[\mathrm{PBE} / \mathrm{AC} / \mathrm{no} S^{2}\right]$} \\
$\mathrm{d}(\AA)$ & $E_{\text {elst }}^{(1)}$ & $E_{\text {exch }}^{(1)}$ & $E_{\text {IND }}^{(2)}$ & $\delta_{\text {int }}^{\mathrm{HF}}[2]$ & $E_{\mathrm{DISP}}^{(2)}$ & $E_{\text {int }}^{[2]}$ & $E_{\text {int }}^{[2]}+\delta_{\text {int }}^{\mathrm{HF}}[2]$ & $E_{\mathrm{CD}}^{(2)}$ & $E_{\mathrm{POL}}^{(2)}$ \\
\hline 1.3 & -64.91 & 165.03 & -23.17 & -26.24 & -19.59 & 57.35 & 31.11 & -12.69 & -10.48 \\
1.4 & -55.71 & 124.39 & -17.60 & -20.71 & -16.14 & 34.94 & 14.24 & -8.46 & -9.14 \\
1.5 & -47.49 & 93.53 & -13.67 & -16.09 & -13.32 & 19.04 & 2.95 & -5.68 & -8.00 \\
1.6 & -40.45 & 70.20 & -10.86 & -12.38 & -11.02 & 7.87 & -4.51 & -3.84 & -7.02 \\
1.7 & -34.56 & 52.63 & -8.82 & -9.45 & -9.15 & 0.11 & -9.34 & -2.63 & -6.19 \\
1.8 & -29.71 & 39.45 & -7.30 & -7.18 & -7.62 & -5.18 & -12.36 & -1.82 & -5.48 \\
1.9 & -25.72 & 29.56 & -6.16 & -5.44 & -6.36 & -8.68 & -14.12 & -1.29 & -4.87 \\
2.0 & -22.46 & 22.17 & -5.27 & -4.11 & -5.33 & -10.89 & -15.00 & -0.93 & -4.34 \\
2.1 & -19.78 & 16.64 & -4.56 & -3.10 & -4.48 & -12.18 & -15.28 & -0.69 & -3.87 \\
2.2 & -17.57 & 12.50 & -3.98 & -2.33 & -3.77 & -12.82 & -15.15 & -0.52 & -3.46 \\
2.3 & -15.73 & 9.41 & -3.49 & -1.76 & -3.18 & -13.01 & -14.77 & -0.39 & -3.10 \\
2.8 & -10.03 & 2.32 & -1.94 & -0.43 & -1.39 & -11.04 & -11.48 & -0.10 & -1.84 \\
3.3 & -7.17 & 0.60 & -1.14 & -0.11 & -0.63 & -8.33 & -8.44 & -0.02 & -1.11 \\
3.8 & -5.46 & 0.16 & -0.69 & -0.03 & -0.29 & -6.29 & -6.32 & -0.01 & -0.68 \\
4.3 & -4.33 & 0.05 & -0.44 & -0.01 & -0.14 & -4.87 & -4.88 & -0.01 & -0.43 \\
4.8 & -3.52 & 0.01 & -0.29 & -0.00 & -0.07 & -3.87 & -3.88 & -0.01 & -0.28 \\
5.3 & -2.92 & 0.00 & -0.20 & -0.00 & -0.04 & -3.16 & -3.16 & -0.01 & -0.20 \\
\hline \hline
\end{tabular}


$7.5 \mathrm{OH}^{-} \ldots \mathrm{H}_{2} \mathrm{O}$

\begin{tabular}{rrrrr}
\hline \hline \multicolumn{5}{c}{$E_{\text {int }}$} \\
\hline $\mathrm{d}(\AA)$ & $\mathrm{CCSD}(\mathrm{T})$ & B3LYP & PBE0 & $\omega$ B97X-D \\
\hline 0.66 & 183.94 & 181.41 & 178.23 & 180.09 \\
0.76 & 80.55 & 79.03 & 75.36 & 77.23 \\
0.86 & 24.31 & 23.29 & 19.48 & 21.40 \\
0.96 & -6.36 & -7.16 & -10.87 & -8.88 \\
1.06 & -22.86 & -23.57 & -27.03 & -25.02 \\
1.16 & -31.31 & -32.00 & -35.12 & -33.17 \\
1.26 & -35.11 & -35.82 & -38.56 & -36.73 \\
1.36 & -36.20 & -36.95 & -39.30 & -37.65 \\
1.46 & -35.72 & -36.49 & -38.48 & -37.06 \\
1.56 & -34.35 & -35.12 & -36.80 & -35.60 \\
2.06 & -24.14 & -24.82 & -25.52 & -24.98 \\
2.56 & -16.05 & -16.62 & -16.95 & -16.50 \\
3.06 & -10.96 & -11.45 & -11.63 & -11.19 \\
3.56 & -7.81 & -8.24 & -8.33 & -7.97 \\
4.06 & -5.81 & -6.18 & -6.21 & -5.94 \\
4.56 & -4.48 & -4.81 & -4.79 & -4.59 \\
5.06 & -3.56 & -3.86 & -3.81 & -3.65 \\
\hline \hline
\end{tabular}




\begin{tabular}{|c|c|c|c|c|c|c|c|c|}
\hline \multirow[b]{2}{*}{$\mathrm{d}(\AA)$} & \multicolumn{4}{|c|}{ PBE0||ALMO } & \multicolumn{4}{|c|}{$\omega B 97 X-D \| A L M O$} \\
\hline & $E_{\text {elst }}+E_{\text {exch }}$ & $E_{\mathrm{pol}}$ & $E_{\mathrm{cd}}$ & $E_{\text {int }}$ & $E_{\text {elst }}+E_{\text {exch }}$ & $E_{\mathrm{pol}}$ & $E_{\text {cd }}$ & $E_{\text {int }}$ \\
\hline 0.66 & 461.68 & -238.26 & -45.18 & 178.23 & 465.44 & -240.33 & -45.02 & 180.09 \\
\hline 0.76 & 295.17 & -177.10 & -42.71 & 75.36 & 298.77 & -178.89 & -42.65 & 77.23 \\
\hline 0.86 & 188.69 & -129.98 & -39.23 & 19.48 & 191.90 & -131.34 & -39.16 & 21.40 \\
\hline 0.96 & 119.13 & -94.98 & -35.01 & -10.87 & 121.85 & -95.87 & -34.85 & -8.88 \\
\hline 1.06 & 73.06 & -69.59 & -30.50 & -27.03 & 75.29 & -70.04 & -30.26 & -25.02 \\
\hline 1.16 & 42.29 & -51.40 & -26.01 & -35.12 & 44.07 & -51.55 & -25.69 & -33.17 \\
\hline 1.26 & 21.67 & -38.41 & -21.82 & -38.56 & 23.06 & -38.38 & -21.41 & -36.73 \\
\hline 1.36 & 7.90 & -29.07 & -18.12 & -39.30 & 8.97 & -28.98 & -17.64 & -37.65 \\
\hline 1.46 & -1.19 & -22.31 & -14.98 & -38.48 & -0.39 & -22.20 & -14.47 & -37.06 \\
\hline 1.56 & -7.06 & -17.36 & -12.38 & -36.80 & -6.46 & -17.27 & -11.87 & -35.60 \\
\hline 2.06 & -14.35 & -6.15 & -5.03 & -25.52 & -14.20 & -6.08 & -4.70 & -24.98 \\
\hline 2.56 & -11.71 & -3.01 & -2.23 & -16.95 & -11.62 & -2.93 & -1.94 & -16.50 \\
\hline 3.06 & -8.80 & -1.74 & -1.09 & -11.63 & -8.73 & -1.69 & -0.77 & -11.19 \\
\hline 3.56 & -6.64 & -1.06 & -0.63 & -8.33 & -6.61 & -1.04 & -0.32 & -7.97 \\
\hline 4.06 & -5.13 & -0.67 & -0.41 & -6.21 & -5.11 & -0.66 & -0.17 & -5.94 \\
\hline 4.56 & -4.05 & -0.44 & -0.30 & -4.79 & -4.05 & -0.44 & -0.10 & -4.59 \\
\hline 5.06 & -3.28 & -0.30 & -0.23 & -3.81 & -3.30 & -0.30 & -0.06 & -3.65 \\
\hline
\end{tabular}

SAPT2

\begin{tabular}{rrrrrrrrrr}
\hline $\mathrm{d}(\AA)$ & $E_{\text {elst }}^{(1)}$ & $E_{\text {exch }}^{(1)}$ & $E_{\text {IND }}^{(2)}$ & $\delta_{\text {int }}^{\mathrm{HF}}[2]$ & $E_{\mathrm{DISP}}^{(2)}$ & $E_{\text {int }}^{[2]}$ & $E_{\text {int }}^{[2]}+\delta_{\text {int }}^{\mathrm{HF}}[2]$ & $E_{\mathrm{CD}}^{(2)}$ \\
\hline 0.66 & -31.21 & 447.53 & -339.88 & 85.42 & -54.87 & 21.57 & 106.99 & -64.32 \\
0.76 & -80.25 & 353.06 & -216.43 & 14.44 & -42.59 & 13.79 & 28.23 & -50.77 \\
0.86 & -94.03 & 274.73 & -140.78 & -17.31 & -33.02 & 6.91 & -10.40 & -39.53 \\
0.96 & -92.04 & 211.52 & -94.37 & -28.35 & -25.70 & -0.58 & -28.94 & -30.07 \\
1.06 & -83.76 & 161.62 & -65.58 & -29.43 & -20.14 & -7.86 & -37.29 & -22.45 \\
1.16 & -73.69 & 122.86 & -47.33 & -26.32 & -15.90 & -14.07 & -40.39 & -16.65 \\
1.26 & -63.84 & 93.11 & -35.43 & -21.90 & -12.66 & -18.83 & -40.73 & -12.41 \\
1.36 & -55.01 & 70.45 & -27.41 & -17.50 & -10.16 & -22.14 & -39.64 & -9.39 \\
1.46 & -47.42 & 53.28 & -21.81 & -13.66 & -8.21 & -24.15 & -37.81 & -7.24 \\
1.56 & -41.02 & 40.33 & -17.74 & -10.50 & -6.67 & -25.10 & -35.61 & -5.69 \\
2.06 & -21.80 & 10.33 & -7.73 & -2.61 & -2.55 & -21.75 & -24.36 & -1.97 \\
2.56 & -13.50 & 2.86 & -3.92 & -0.64 & -1.06 & -15.61 & -16.26 & -0.73 \\
3.06 & -9.27 & 0.86 & -2.12 & -0.16 & -0.47 & -11.00 & -11.16 & -0.29 \\
3.56 & -6.79 & 0.28 & -1.22 & -0.04 & -0.21 & -7.95 & -7.99 & -0.12 \\
4.06 & -5.20 & 0.09 & -0.74 & -0.01 & -0.10 & -5.95 & -5.96 & -0.05 \\
4.56 & -4.12 & 0.03 & -0.47 & -0.00 & -0.05 & -4.61 & -4.61 & -0.02 \\
5.06 & -3.34 & 0.01 & -0.32 & 0.00 & -0.03 & -3.67 & -3.67 & -0.01 \\
\hline \hline
\end{tabular}




\begin{tabular}{rrrrrrrrrr}
\hline \hline \multicolumn{7}{c}{ SAPT2+3 } \\
\hline $\mathrm{d}(\AA)$ & $E_{\text {elst }}^{(1)}$ & $E_{\text {exch }}^{(1)}$ & $E_{\text {IND }}^{[3]}$ & $\delta_{\text {int }}^{\mathrm{HF}}[3]$ & $E_{\text {DISP }}^{[3]}$ & $E_{\text {int }}^{[3]}$ & $E_{\text {int }}^{[3]}+\delta_{\text {int }}^{\text {HF }}[3]$ & $E_{\text {CD }}^{[3]}$ \\
\hline 0.66 & -40.19 & 447.53 & -761.43 & 506.97 & -73.82 & -427.90 & 79.07 & -230.06 \\
0.76 & -87.03 & 353.06 & -459.53 & 257.54 & -54.63 & -248.13 & 9.41 & -172.25 \\
0.86 & -98.99 & 274.73 & -281.49 & 123.41 & -40.69 & -146.44 & -23.03 & -122.77 \\
0.96 & -95.54 & 211.52 & -176.84 & 54.11 & -30.76 & -91.61 & -37.50 & -83.80 \\
1.06 & -86.13 & 161.62 & -114.91 & 19.90 & -23.70 & -63.12 & -43.23 & -55.90 \\
1.16 & -75.22 & 122.86 & -77.59 & 3.95 & -18.61 & -48.57 & -44.62 & -37.18 \\
1.26 & -64.75 & 93.11 & -54.50 & -2.83 & -14.87 & -41.02 & -43.85 & -25.05 \\
1.36 & -55.48 & 70.45 & -39.74 & -5.17 & -12.06 & -36.83 & -42.01 & -17.23 \\
1.46 & -47.57 & 53.28 & -29.97 & -5.50 & -9.89 & -34.15 & -39.65 & -12.13 \\
1.56 & -40.96 & 40.33 & -23.25 & -5.00 & -8.19 & -32.06 & -37.05 & -8.73 \\
2.06 & -21.44 & 10.33 & -8.62 & -1.72 & -3.43 & -23.15 & -24.87 & -2.35 \\
2.56 & -13.22 & 2.86 & -4.06 & -0.50 & -1.52 & -15.93 & -16.43 & -1.18 \\
3.06 & -9.08 & 0.86 & -2.14 & -0.15 & -0.70 & -11.05 & -11.20 & -0.30 \\
3.56 & -6.67 & 0.28 & -1.21 & -0.05 & -0.33 & -7.93 & -7.97 & -0.13 \\
4.06 & -5.12 & 0.09 & -0.73 & -0.02 & -0.16 & -5.91 & -5.93 & -0.05 \\
4.56 & -4.06 & 0.03 & -0.47 & -0.01 & -0.08 & -4.57 & -4.58 & -0.02 \\
5.06 & -3.30 & 0.01 & -0.31 & -0.00 & -0.04 & -3.64 & -3.64 & -0.01 \\
\hline \hline
\end{tabular}

\begin{tabular}{rrrrrrrrrrr}
\hline \hline \multicolumn{10}{c}{$\mathrm{SAPT}(\mathrm{DFT})\left[\mathrm{PBE} 0 / \mathrm{AC} / S^{2}\right]$} \\
\hline $\mathrm{d}(\AA)$ & $E_{\text {elst }}^{(1)}$ & $E_{\text {exch }}^{(1)}$ & $E_{\text {IND }}^{(2)}$ & $\delta_{\text {int }}^{\mathrm{HF}}[2]$ & $E_{\text {DISP }}^{(2)}$ & $E_{\text {int }}^{[2]}$ & $E_{\text {int }}^{[2]}+\delta_{\text {int }}^{\mathrm{HF}}[2]$ & $E_{\mathrm{CD}}^{(2)}$ & $E_{\text {POL }}^{(2)}$ \\
\hline 0.66 & -41.23 & 348.09 & -322.30 & 190.40 & -46.20 & -61.63 & 128.76 & -312.43 & -9.87 \\
0.76 & -87.59 & 285.75 & -204.31 & 82.43 & -36.87 & -43.01 & 39.42 & -186.91 & -17.39 \\
0.86 & -99.20 & 229.84 & -131.75 & 25.57 & -29.42 & -30.53 & -4.96 & -111.61 & -20.14 \\
0.96 & -95.53 & 181.73 & -87.20 & -1.88 & -23.59 & -24.59 & -26.47 & -66.78 & -20.41 \\
1.06 & -86.00 & 141.76 & -59.58 & -13.38 & -19.04 & -22.86 & -36.24 & -40.19 & -19.39 \\
1.16 & -75.01 & 109.47 & -42.15 & -16.72 & -15.49 & -23.18 & -39.90 & -24.39 & -17.75 \\
1.26 & -64.50 & 83.90 & -30.93 & -16.20 & -12.69 & -24.22 & -40.42 & -15.00 & -15.94 \\
1.36 & -55.22 & 63.99 & -23.55 & -14.11 & -10.46 & -25.23 & -39.34 & -9.41 & -14.14 \\
1.46 & -47.31 & 48.67 & -18.52 & -11.63 & -8.67 & -25.84 & -37.47 & -6.06 & -12.47 \\
1.56 & -40.71 & 36.96 & -14.97 & -9.28 & -7.23 & -25.94 & -35.22 & -4.00 & -10.96 \\
2.06 & -21.28 & 9.49 & -6.58 & -2.47 & -3.07 & -21.44 & -23.91 & -0.79 & -5.79 \\
2.56 & -13.11 & 2.63 & -3.53 & -0.54 & -1.38 & -15.39 & -15.94 & -0.32 & -3.20 \\
3.06 & -9.01 & 0.80 & -2.04 & -0.06 & -0.64 & -10.90 & -10.95 & -0.21 & -1.84 \\
3.56 & -6.62 & 0.27 & -1.22 & 0.02 & -0.31 & -7.88 & -7.85 & -0.13 & -1.09 \\
4.06 & -5.08 & 0.09 & -0.77 & 0.04 & -0.15 & -5.90 & -5.87 & -0.10 & -0.66 \\
4.56 & -4.03 & 0.03 & -0.51 & 0.03 & -0.08 & -4.58 & -4.55 & -0.09 & -0.43 \\
5.06 & -3.27 & 0.01 & -0.36 & 0.03 & -0.04 & -3.66 & -3.64 & -0.07 & -0.29 \\
\hline \hline
\end{tabular}




\begin{tabular}{rrrrrrrrrr}
\hline \hline \multicolumn{10}{c}{$\mathrm{SAPT}(\mathrm{DFT})\left[\mathrm{PBE} / \mathrm{AC} / \mathrm{nO} S^{2}\right]$} \\
\hline $\mathrm{d}(\AA)$ & $E_{\text {elst }}^{(1)}$ & $E_{\text {exch }}^{(1)}$ & $E_{\text {IND }}^{(2)}$ & $\delta_{\text {int }}^{\mathrm{HF}}[2]$ & $E_{\mathrm{DISP}}^{(2)}$ & $E_{\text {int }}^{[2]}$ & $E_{\text {int }}^{[2]}+\delta_{\text {int }}^{\mathrm{HF}}[2]$ & $E_{\mathrm{CD}}^{(2)}$ & $E_{\mathrm{POL}}^{(2)}$ \\
\hline 0.66 & -41.23 & 455.37 & -135.20 & -88.88 & -46.20 & 232.74 & 143.86 & -106.70 & -28.50 \\
0.76 & -87.59 & 355.93 & -97.06 & -82.90 & -36.87 & 134.41 & 51.51 & -69.69 & -27.37 \\
0.86 & -99.20 & 274.41 & -70.83 & -70.94 & -29.42 & 74.97 & 4.02 & -45.06 & -25.76 \\
0.96 & -95.53 & 209.40 & -52.68 & -57.66 & -23.59 & 37.60 & -20.06 & -29.07 & -23.60 \\
1.06 & -86.00 & 158.64 & -39.99 & -45.40 & -19.04 & 13.61 & -31.79 & -18.84 & -21.16 \\
1.16 & -75.01 & 119.64 & -31.00 & -35.03 & -15.49 & -1.85 & -36.88 & -12.30 & -18.70 \\
1.26 & -64.50 & 89.99 & -24.52 & -26.68 & -12.69 & -11.73 & -38.41 & -8.11 & -16.41 \\
1.36 & -55.22 & 67.61 & -19.78 & -20.15 & -10.46 & -17.84 & -37.99 & -5.42 & -14.35 \\
1.46 & -47.31 & 50.81 & -16.23 & -15.14 & -8.67 & -21.40 & -36.55 & -3.68 & -12.55 \\
1.56 & -40.71 & 38.23 & -13.52 & -11.35 & -7.23 & -23.23 & -34.58 & -2.55 & -10.98 \\
2.06 & -21.28 & 9.59 & -6.31 & -2.67 & -3.07 & -21.07 & -23.74 & -0.54 & -5.77 \\
2.56 & -13.11 & 2.64 & -3.34 & -0.65 & -1.38 & -15.19 & -15.84 & -0.14 & -3.20 \\
3.06 & -9.01 & 0.80 & -1.88 & -0.16 & -0.64 & -10.73 & -10.89 & -0.05 & -1.83 \\
3.56 & -6.62 & 0.27 & -1.11 & -0.04 & -0.31 & -7.77 & -7.81 & -0.03 & -1.09 \\
4.06 & -5.08 & 0.09 & -0.69 & -0.01 & -0.15 & -5.83 & -5.84 & -0.03 & -0.66 \\
4.56 & -4.03 & 0.03 & -0.45 & -0.00 & -0.08 & -4.52 & -4.52 & -0.02 & -0.43 \\
5.06 & -3.27 & 0.01 & -0.30 & 0.00 & -0.04 & -3.61 & -3.61 & -0.02 & -0.29 \\
\hline \hline
\end{tabular}

\section{Complexes cartesian coordinates}

\section{$8.1 \quad\left(\mathrm{H}_{2} \mathrm{O}\right)_{2}$}

Dimer1 $\mathrm{R}(\mathrm{O} . . \mathrm{H})=1.54 \AA$

O 0.0000000 .0000000 .000000

H $-0.7690000 .000000-0.594000$

H $0.7690000 .000000-0.594000$

O $-2.1370000 .000000-1.298000$

H -2.689000 $0.769000-1.080000$

H -2.689000 - $0.769000-1.080000$

Dimer2 $\mathrm{R}(\mathrm{O} . . \mathrm{H})=1.74 \AA$ 
O 0.0000000 .0000000 .000000

H -0.769000 $0.000000-0.594000$

H $0.7690000 .000000-0.594000$

O -2.308000 $0.000000-1.401000$

H -2.860000 $0.769000-1.183000$

H -2.860000 -0.769000 -1.183000

Dimer3 $\mathrm{R}(\mathrm{O} . . \mathrm{H})=1.84 \AA$

O 0.0000000 .0000000 .000000

H -0.769000 $0.000000-0.594000$

H $0.7690000 .000000-0.594000$

O -2.393000 $0.000000-1.453000$

H -2.945000 $0.769000-1.235000$

H -2.945000 -0.769000 -1.235000

Dimer4 R $(\mathrm{O} . . \mathrm{H})=1.94 \AA$

O 0.0000000 .0000000 .000000

H -0.769000 $0.000000-0.594000$

H $0.7690000 .000000-0.594000$

O -2.479000 $0.000000-1.505000$

H -3.031000 $0.769000-1.287000$

H -3.031000 -0.769000 -1.287000

Dimer5 R $(\mathrm{O} . . \mathrm{H})=2.04 \AA$ 
O 0.0000000 .0000000 .000000

H -0.769000 $0.000000-0.594000$

H $0.7690000 .000000-0.594000$

O $-2.5580000 .000000-1.553000$

H -3.110000 $0.769000-1.335000$

H -3.110000 -0.769000 -1.335000

Dimer6 R $(\mathrm{O} . . \mathrm{H})=2.14 \AA$

O 0.0000000 .0000000 .000000

H -0.769000 $0.000000-0.594000$

H $0.7690000 .000000-0.594000$

O -2.650000 $0.000000-1.609000$

H -3.202000 0.769000 -1.391000

H -3.202000 -0.769000 -1.391000

Dimer7 R $(\mathrm{O} . . \mathrm{H})=2.24 \AA$

O 0.0000000 .0000000 .000000

H -0.769000 $0.000000-0.594000$

H $0.7690000 .000000-0.594000$

O -2.735000 $0.000000-1.661000$

H -3.287000 $0.769000-1.443000$

H -3.287000 -0.769000 -1.443000

Dimer8 R $(\mathrm{O} . . \mathrm{H})=2.34 \AA$ 
O 0.0000000 .0000000 .000000

H -0.769000 $0.000000-0.594000$

H $0.7690000 .000000-0.594000$

O -2.821000 $0.000000-1.713000$

H -3.373000 $0.769000-1.495000$

H -3.373000 -0.769000 -1.495000

Dimer9 $\mathrm{R}(\mathrm{O} . \mathrm{H})=2.54 \AA$

O 0.0000000 .0000000 .000000

H -0.769000 $0.000000-0.594000$

H $0.7690000 .000000-0.594000$

O -2.992000 $0.000000-1.817000$

H -3.544000 $0.769000-1.599000$

H -3.544000 -0.769000 -1.599000

Dimer10 R(O..H $)=3.04 \AA$

O 0.0000000 .0000000 .000000

H -0.769000 $0.000000-0.594000$

H $0.7690000 .000000-0.594000$

O -3.419000 $0.000000-2.076000$

H -3.971000 $0.769000-1.858000$

H -3.971000 -0.769000 -1.858000

Dimer11 R(O..H $)=4.04 \AA$ 
O 0.0000000 .0000000 .000000

H -0.769000 $0.000000-0.594000$

H $0.7690000 .000000-0.594000$

O $-4.2740000 .000000-2.595000$

H -4.826000 $0.769000-2.377000$

H -4.826000 -0.769000 -2.377000

Dimer12 R(O..H $)=5.04 \AA$

O 0.0000000 .0000000 .000000

H -0.769000 $0.000000-0.594000$

H $0.7690000 .000000-0.594000$

O $-5.1290000 .000000-3.114000$

H -5.681000 $0.769000-2.896000$

H $-5.681000-0.769000-2.896000$

\subsection{Ions $\ldots \mathrm{H}_{2} \mathrm{O}$}

1. $\mathrm{Zn}^{2+} \ldots \mathrm{H}_{2} \mathrm{O}: \mathrm{R}_{e q}\left(\mathrm{Zn}^{2+} . . \mathrm{O}\right)=1.9 \AA$

Zn 1.90000 .00 .0

O 0.0000000 .0000000 .000000

H -0.559244 0.7768390 .000000

H $-0.559244-0.7768390 .000000$

Anions-water complexes geometries from ref ${ }^{1}$ 
2. $\mathrm{F}^{-} \ldots \mathrm{H}_{2} \mathrm{O}: \mathrm{R}_{e q}\left(\mathrm{~F}^{-} . . \mathrm{H}\right)=1.37 \AA$

F -1.2363890.0122390.000000

O $1.197566-0.1080870 .000000$

H 1.4153970 .8270140 .000000

H $0.134830-0.0843780 .000000$

3. $\mathrm{Cl}^{-} \ldots \mathrm{H}_{2} \mathrm{O}: \mathrm{R}_{e q}\left(\mathrm{Cl}^{-} . . \mathrm{H}\right)=2.12 \AA$

Cl -1.351043 0.035294 0.000000

O $1.741354-0.0865720 .000000$

H 1.8290900 .8701300 .000000

H $0.754930-0.1794000 .000000$

4. $\mathrm{OH}^{-} \ldots \mathrm{H}_{2} \mathrm{O}: \operatorname{Req}\left(\mathrm{OH}^{-} . . \mathrm{H}\right)=1.36 \AA$

O $1.23583426-0.09177426-0.06793615$

H 1.547558310 .571928350 .55691305

O -1.23110973 0.09790429 -0.05372440

H -0.11773792 $0.03366878-0.06528348$

H -1.46896962 -0.65591986 0.49310314 


\section{References}

(1) Lao, K. U.; Schäffer, R.; Jansen, G.; Herbert, J. M. Accurate Description of Intermolecular Interactions Involving Ions Using Symmetry-Adapted Perturbation Theory. J. Chem. Theory Comput. 2015, 11, 2473-2486.

(2) Lee, D; Burke, K. Finding Electron Affinities with Approximate Density Functionals. Mol. Phys. 2010, 108, 2687-2701.

(3) Garmer, D. R.; Gresh, N. A comprehensive energy component analysis of the interaction of hard and soft dications with biological ligands. J. Am. Chem. Soc. 1994, 116, 35563567.

(4) Gresh, N.; Stevens, W. J.; Krauss, M. Mono-and poly-ligated complexes of Zn2+: An ab initio analysis of the metal-ligand interaction energy. J. Comput. Chem. 1995, 16, $843-855$.

(5) Gresh, N. Energetics of Zn2+ binding to a series of biologically relevant ligands: A molecular mechanics investigation grounded on ab initio self-consistent field supermolecular computations. J. Comput. Chem. 1995, 16, 856-882.

(6) Gresh, N.; Garmer, D. R. Comparative binding energetics of Mg2+, Ca2+, Zn2+, and $\mathrm{Cd} 2+$ to biologically relevant ligands: combined ab initio SCF supermolecule and molecular mechanics investigation. J. Comput. Chem. 1996, 17, 1481-1495.

(7) Gourlaouen, C.; Clavaguéra, C.; Marjolin, A.; Piquemal, J.-P.; Dognon, J.-P. Understanding the structure and electronic properties of Th4+-water complexes. Can. J. Chem. 2013, 91, 821-831.

(8) Gilmore, R. A. J.; Dove, M. T.; Misquitta, A. J. First-Principles Many-Body Nonadditive Polarization Energies from Monomer and Dimer Calculations Only: A Case Study on Water. J. Chem. Theory Comput. 2020, 16, 224-242. 
(9) Tang, K. T.; Toennies, J. P. An improved simple model for the van der Waals potential based on universal damping functions for the dispersion coefficients. J. Chem. Phys. 1984, 80, 3726-3741.

(10) Misquitta, A. J.; Stone, A. J.; Fazeli, F. Distributed Multipoles from a Robust BasisSpace Implementation of the Iterated Stockholder Atoms Procedure. J. Chem. Theory Comput. 2014, 10, 5405-5418.

(11) Misquitta, A. J.; Stone, A. J. ISA-Pol: Distributed Polarizabilities and Dispersion Models from a Basis-Space Implementation of the Iterated Stockholder Atoms Procedure. Theor. Chem. Acc. 2018, 137, 153.

(12) Misquitta, A.; Stone, A. CamCASP: a program for studying intermolecular interactions and for the calculation of molecular properties in distributed form. University of Cambridge 2018,

(13) Misquitta, A. J.; Stone, A. J. Ab Initio Atom-Atom Potentials Using CamCASP: Theory and Application to Many-Body Models for the Pyridine Dimer. J. Chem. Theory Comput. 2016, 12, 4184-4208.

(14) Misquitta, A. J.; Podeszwa, R.; Jeziorski, B.; Szalewicz, K. Intermolecular Potentials Based on Symmetry-Adapted Perturbation Theory with Dispersion Energies from Time-Dependent Density-Fun ctional Calculations. J. Chem. Phys. 2005, 123, 214103.

(15) Heßelmann, A.; Jansen, G. First-order intermolecular interaction energies from Kohn-Sham orbitals. Chem. Phys. Lett. 2002, 357, 464-470.

(16) Heßelmann, A.; Jansen, G. Intermolecular induction and exchange-induction energies from coupled-perturbed Kohn-Sham density functional theory. Chem. Phys. Lett. 2002, 362, 319-325. 
(17) Heßelmann, A.; Jansen, G. Intermolecular dispersion energies from time-dependent density functional theory. Chem. Phys. Lett. 2003, 367, 778-784.

(18) Misquitta, A. J.; Szalewicz, K. Intermolecular forces from asymptotically corrected density functional description of monomers. Chem. Phys. Lett. 2002, 357, 301-306.

(19) Misquitta, A. J.; Jeziorski, B.; Szalewicz, K. Dispersion Energy from Density-Functional Theory Description of Monomers. Phys. Rev. Lett. 2003, 91, 033201.

(20) Cencek, W.; Szalewicz, K. On Asymptotic Behavior of Density Functional Theory. J. Chem. Phys. 2013, 139, 024104-024104-27.

(21) Misquitta, A. J. Charge-transfer from Regularized Symmetry-Adapted Perturbation Theory. J. Chem. Theory Comput. 2013, 9, 5313-5326.

(22) Williams, H. L.; Mas, E. M.; Szalewicz, K.; Jeziorski, B. On the effectiveness of monomer-, dimer-, and bond-centered basis functions in calculations of intermolecular interaction energies. J. Chem. Phys. 1995, 103, 7374-7391.

(23) Podeszwa, P.; Bukowski, R.; Szalewicz, K. Potential energy surface for the benzene dimer and perturbational analysis of $\pi-\pi$ interactions. J. Phys. Chem. A 2006, 110, $10345-10354$. 